\title{
(T)-structures over two-dimensional F-manifolds: formal classification
}

\author{
Liana David $^{1}$ (D) Claus Hertling ${ }^{2}$
}

Received: 2 December 2018 / Accepted: 28 September 2019 / Published online: 14 October 2019 (c) Fondazione Annali di Matematica Pura ed Applicata and Springer-Verlag GmbH Germany, part of Springer Nature 2019

\begin{abstract}
A $(T E)$-structure $\nabla$ over a complex manifold $M$ is a meromorphic connection defined on a holomorphic vector bundle over $\mathbb{C} \times M$, with poles of Poincaré rank one along $\{0\} \times M$. Under a mild additional condition (the so-called unfolding condition), $\nabla$ induces a multiplication on $T M$ and a vector field on $M$ (the Euler field), which make $M$ into an $F$-manifold with Euler field. By taking the pullbacks of $\nabla$ under the inclusions $\{z\} \times M \rightarrow \mathbb{C} \times M\left(z \in \mathbb{C}^{*}\right)$, we obtain a family of flat connections on vector bundles over $M$, parameterized by $z \in \mathbb{C}^{*}$. The properties of such a family of connections give rise to the notion of $(T)$-structure. Therefore, any $(T E)$-structure underlies a $(T)$-structure, but the converse is not true. The unfolding condition can be defined also for $(T)$-structures. A $(T)$-structure with the unfolding condition induces on its parameter space the structure of an $F$-manifold (without Euler field). After a brief review on the theory of $(T)$ - and $(T E)$-structures, we determine normal forms for the equivalence classes, under formal isomorphisms, of $(T)$-structures which induce a given irreducible germ of two-dimensional $F$-manifolds.
\end{abstract}

Keywords Meromorphic connections $\cdot(\mathrm{T})$ and $(T E)$-structures $\cdot F$-manifolds $\cdot$ Euler fields · Frobenius manifolds · Formal classifications

Mathematics Subject Classification 53B15 $\cdot 35 \mathrm{~J} 99 \cdot 32 \mathrm{~A} 20 \cdot 53 \mathrm{~B} 50$

\section{Introduction}

The theory of meromorphic connections is a well-established field with importance in many areas of modern mathematics (complex analysis, algebraic geometry, differential geometry, integrable systems, etc.). An important class of meromorphic connections are the so-called

Liana David

liana.david@imar.ro

Claus Hertling

hertling@math.uni-mannheim.de

1 "Simion Stoilow" Institute of Mathematics of the Romanian Academy, Calea Grivitei 21, Sector 1, Bucharest, Romania

2 Lehrstuhl für Mathematik, Universität Mannheim, B6, 26, 68131, Mannheim, Germany 
$(T E)$-structures. They are meromorphic connections defined on holomorphic vector bundles over products $\mathbb{C} \times M$, with poles of Poincaré rank one along the submanifold $\{0\} \times M$. They represent the simplest class of meromorphic connections with irregular singularities. The parameter space $M$ of a $(T E)$-structure inherits, under a mild additional condition (the 'unfolding condition') a multiplication $\circ$ on $T M$, with nice properties (fiber-preserving, commutative, associative, with unit field, and satisfying a certain integrability condition), and a vector field $E$ which rescales $\circ$, making $M$ into a so-called $F$-manifold with Euler field. The notion of an $F$-manifold was introduced for the first time in [10] as a generalization of the notion of a Frobenius manifold [5]. Any Frobenius manifold without metric is an $F$ manifold. As shown in [12], there are $F$-manifolds which cannot be enriched to a Frobenius manifold. Examples of $F$-manifolds arise also in the theory of integrable systems [14,19] and quantum cohomology [12].

A natural question which arises in this context is to classify the $(T E)$-structures over a given germ of $F$-manifolds with Euler field. While a $(T E)$-structure $\nabla$ may be seen as a family of meromorphic connections on vector bundles over $\Delta$ (a small disk centered at the origin $0 \in \mathbb{C}$ ), by 'forgetting' the derivatives $\nabla_{X}$ where $X \in \mathcal{T}_{M}$ is lifted naturally to $\mathbb{C} \times M$ (this point of view being crucial in the theory of isomondromic deformations), we may adopt the alternative viewpoint and study the derivatives $\nabla_{X}$ as a family of flat connections on vector bundles over $M$ parameterized by $z \in \mathbb{C}^{*}$. Such a family has received much attention in the theory of meromorphic connections and is referred in the literature as a $(T)$-structure over $M$. Therefore, any $(T E)$-structure underlies a $(T)$-structure, but the converse is not always true. The parameter space of a $(T)$-structure inherits the structure of an $F$-manifold (without Euler field), when the unfolding condition is satisfied.

Adopting the second viewpoint, in this paper we make a first step in the classification of $(T E)$-structures over a given germ of $F$-manifolds with Euler field. We consider the simplest case, namely when the germ is two-dimensional and irreducible and we determine formal normal forms for the $(T)$-structures over such germs. The results we prove here will be crucial for future projects, where we shall classify $(T E)$-structures over two-dimensional (and, possibly bigger dimensional) germs of $F$-manifolds with Euler fields. The two-dimensional case is considerably simpler, owing to the fact that (unlike higher dimensions) irreducible germs of two-dimensional $F$-manifolds are classified [9]: either they coincide with the germ of the globally nilpotent constant $F$-manifold $\mathcal{N}_{2}$, or they are generically semisimple and belong to a class of germs $I_{2}(m)$ parameterized by $m \in \mathbb{N}_{\geq 3}$ (see the end of Sect. 2.2 for the description of these germs). As $F$-manifolds isomorphisms lift to isomorphisms between the spaces of $(T)$ and ( $T E$ )-structures over them, we can (and will) assume, without loss of generality, that our germs of $F$-manifolds are $\mathcal{N}_{2}$ or $I_{2}(m)$ (with $m \geq 3$ ). The specific form of these germs will enable us to find the formal normal forms for $(T)$-structures over them.

Structure of the paper In Sect. 2, we recall well-known facts we need on $(T),(T E)$ structures and $F$-manifolds (see, e.g., [8]). Although our original contribution in this paper refers to $(T)$-structures, we include also basic material on $(T E)$-structures as a motivation and to fix notation and results we shall use in the subsequent stages of our project on classification of $(T E)$-structures. In Sect. 3, we study various classes of differential equations which will be relevant in our treatment. In Sect. 4, we determine the formal normal forms for $(T)$ structures over $I_{2}(m)$, and in Sect. 5, we study the similar question for $(T)$-structures over $\mathcal{N}_{2}$. A main difference between these two cases lies in the form of formal isomorphisms used in the classification. The automorphism group of $I_{2}(\mathrm{~m})$ is finite (see Lemma 11) and formal $(T)$-structure isomorphisms which lift non-trivial automorphisms of $I_{2}(m)$ do not add much simplification in the expressions of $(T)$-structures over $I_{2}(m)$. For this reason, in the case of $I_{2}(m)$, we content ourselves to determine formal normal forms for $(T)$-structures 
which are formally gauge isomorphic (i.e., are isomorphic by means of formal isomorphisms which lift the identity map of $I_{2}(m)$ ). This is done in Theorem 16. As opposed to $I_{2}(m)$, the germ $\mathcal{N}_{2}$ has a rich automorphism group (see Lemma 11). The $(T)$-structures over $\mathcal{N}_{2}$ will be classified up to formal gauge isomorphisms in Theorem 18 and up to the entire group of formal isomorphisms in Theorem 21. Formal isomorphisms which lift non-trivial automorphisms of $\mathcal{N}_{2}$ simplify considerably the classification. Their role in the classification is explained in Theorem 19.

\section{Preliminary material}

We begin by fixing our notation.

Notation 1 For a complex manifold $M$, we denote by $\mathcal{O}_{M}, \mathcal{T}_{M}, \Omega_{M}^{k}$ the sheaves of holomorphic functions, holomorphic vector fields and holomorphic $k$-forms on $M$, respectively. For a holomorphic vector bundle $H$, we denote by $\mathcal{O}(H)$ the sheaf of its holomorphic sections. We denote by $\Omega_{\mathbb{C} \times M}^{1}(\log \{0\} \times M)$ the sheaf of meromorphic 1 -forms on $\mathbb{C} \times M$, which are logarithmic along $\{0\} \times M$. Locally, in a neighborhood of $(0, p)$, where $p \in M$, any $\omega \in \Omega_{\mathbb{C} \times M}^{1}(\log \{0\} \times M)$ is of the form

$$
\omega=\frac{f(z, t)}{z} \mathrm{~d} z+\sum_{i} f_{i}(z, t) \mathrm{d} t_{i}
$$

where $\left(t_{i}\right)$ is a coordinate system of $M$ around $p$ and $f, f_{i}$ are holomorphic. The ring of holomorphic functions defined on a neighborhood of $0 \in \mathbb{C}$ will be denoted by $\mathbb{C}\{z\}$, the subring of formal power series $\sum_{n \geq 0} a_{n} z^{n}$ will be denoted by $\mathbb{C}[[z]]$, the subring of power series $\sum_{n \geq 0} a_{n} z^{n}$ with $a_{n}=0$ for any $n \leq k-1$ will be denoted by $\mathbb{C}[[z]]_{\geq k}$, and the vector space of polynomials of degree at most $k$ in the variables $t=\left(t_{i}\right)$ will be denoted by $\mathbb{C}[t]_{\leq k}$. Finally, we denote by $\left.\mathbb{C}\{t, z]\right]$ the ring of formal power series $\sum_{n \geq 0} a_{n} z^{n}$ where all $a_{n}=a_{n}(t)$ are holomorphic on the same neighborhood of $0 \in \mathbb{C}$ and by $\mathbb{C}[[z]][t]_{\leq k}$ the vector space of formal power series $\sum_{n \geq 0} a_{n} z^{n}$ with $a_{n}$ polynomials of degree at most $k$ in $t$. For a function $f \in \mathbb{C}\{t, z]]$ and matrix $\left.A \in M_{k \times k}(\mathbb{C}\{t, z]]\right)$, we often write $f=\sum_{n \geq 0} f(n) z^{n}$ and $A=\sum_{n \geq 0} A(n) z^{n}$ where $f(n) \in \mathbb{C}\{t\}$ and $A(n) \in M_{k \times k}(\mathbb{C}\{t\})$.

\section{$2.1(T)$ and $(T E)$-structures}

In this section, we recall basic facts on $(T)$ and $(T E)$-structures.

Definition 2 Let $M$ be a complex manifold and $H \rightarrow \mathbb{C} \times M$ a holomorphic vector bundle.

(i) $[11$, Def. 3.1] A $(T)$-structure over $M$ is a pair $(H \rightarrow \mathbb{C} \times M, \nabla)$ where $\nabla$ is a map

$$
\nabla: \mathcal{O}(H) \rightarrow \frac{1}{z} \mathcal{O}_{\mathbb{C} \times M} \cdot \Omega_{M}^{1} \otimes \mathcal{O}(H)
$$

such that, for any $z \in \mathbb{C}^{*}$, the restriction of $\nabla$ to $\left.H\right|_{\{z\} \times M}$ is a flat connection.

(ii) $[11$, Def. 2.1] A $(T E)$-structure over $M$ is a pair $(H \rightarrow \mathbb{C} \times M, \nabla)$ where $\nabla$ is a flat connection on $\left.H\right|_{\mathbb{C}^{*} \times M}$ with poles of Poincaré rank 1 along $\{0\} \times M$ :

$$
\nabla: \mathcal{O}(H) \rightarrow \frac{1}{z} \Omega_{\mathbb{C} \times M}^{1}(\log (\{0\} \times M) \otimes \mathcal{O}(H) .
$$


Any ( $T E$ )-structure determines (by forgetting the derivative in the $z$ direction) a $(T)$ structure ('E' comes from extension).

Let $(H \rightarrow \mathbb{C} \times M, \nabla)$ be a $(T E)$-structure and $\Delta \subset \mathbb{C}$ a small disk centered at the origin, $U \subset M$ a coordinate chart with coordinates $\left(t_{1}, \ldots, t_{m}\right)$, such that $\left.H\right|_{\Delta \times U}$ is trivial. Using a trivialization $\underline{s}=\left(s_{1}, \ldots, s_{r}\right)$ of $\left.H\right|_{\Delta \times U}$, we write

$$
\begin{aligned}
\nabla\left(s_{i}\right) & =\sum_{j=1}^{r} \Omega_{j i} s_{j}, \quad \text { short : } \quad \nabla(\underline{s})=\underline{s} \cdot \Omega, \\
\Omega & =\sum_{i=1}^{m} z^{-1} A_{i}(z, t) \mathrm{d} t_{i}+z^{-2} B(z, t) \mathrm{d} z,
\end{aligned}
$$

where $A_{i}, B$ are holomorphic,

$$
A_{i}(z, t)=\sum_{k \geq 0} A_{i}(k) z^{k}, \quad B(z, t)=\sum_{k \geq 0} B(k) z^{k}
$$

and $A_{i}(k)$ and $B(k)$ depend only on $t \in U$. The flatness of $\nabla$ gives, for any $i \neq j$,

$$
\begin{aligned}
& z \partial_{i} A_{j}-z \partial_{j} A_{i}+\left[A_{i}, A_{j}\right]=0, \\
& z \partial_{i} B-z^{2} \partial_{z} A_{i}+z A_{i}+\left[A_{i}, B\right]=0 .
\end{aligned}
$$

[when $\nabla$ is a $(T)$-structure, the summand $z^{-2} B(t, z) \mathrm{d} z$ in $\Omega$ and relations (6) are dropped]. Relations (5), (6) split according to the powers of $z$ as follows: for any $k \geq 0$,

$$
\begin{aligned}
& \partial_{i} A_{j}(k-1)-\partial_{j} A_{i}(k-1)+\sum_{l=0}^{k}\left[A_{i}(l), A_{j}(k-l)\right]=0, \\
& \partial_{i} B(k-1)-(k-2) A_{i}(k-1)+\sum_{l=0}^{k}\left[A_{i}(l), B(k-l)\right]=0,
\end{aligned}
$$

where $A_{i}(-1)=B(-1)=0$.

Definition 3 (i) An isomorphism $T:(\tilde{H}, \tilde{\nabla}) \rightarrow(H, \nabla)$ between two $(T)$-structures over $\tilde{M}$ and $M$, respectively, is a holomorphic vector bundle isomorphism $T: \tilde{H} \rightarrow H$ which covers a biholomorphic map of the form $\mathrm{Id} \times h: \mathbb{C} \times \tilde{M} \rightarrow \mathbb{C} \times M$, i.e., $T\left(\tilde{H}_{(z, \tilde{p})}\right) \subset H_{(z, h(\tilde{p}))}$, for any $\tilde{p} \in \tilde{M}$, and is compatible with connections:

$$
T\left(\tilde{\nabla}_{X_{\tilde{p}}}(s)\right)=\nabla_{h_{*}\left(X_{\tilde{p})}\right.}(T(s)), \quad \forall X_{\tilde{p}} \in T_{\tilde{p}} \tilde{M}, \quad \tilde{p} \in \tilde{M}, s \in \mathcal{O}(\tilde{H}) .
$$

Above, $T(s) \in \mathcal{O}(H)$ is defined by $T(s)_{(z, p)}:=T\left(s_{\left(z, h^{-1}(p)\right)}\right)$, for any $p \in M$.

(ii) An isomorphism $T:(\tilde{H}, \tilde{\nabla}) \rightarrow(H, \nabla)$ between two $(T E)$-structures is an isomorphism between their underlying $(T)$-structures, which satisfies in addition

$$
T\left(\tilde{\nabla}_{\partial_{z}}(s)\right)=\nabla_{\partial_{z}}(T(s)), \quad \forall s \in \mathcal{O}(\tilde{H}) .
$$

Recall that if $f: \tilde{N} \rightarrow N$ is a map and $\pi: E \rightarrow N$ is a bundle over $N$, then $f^{*} E:=$ $\{(e, \tilde{p}) \in E \times \tilde{N}, \pi(e)=f(\tilde{p})\}$ is a bundle over $\tilde{N}$ with bundle projection $(e, \tilde{p}) \rightarrow \tilde{p}$. Any section $s \in \mathcal{O}(E)$ defines a section $f^{*} s \in \mathcal{O}\left(f^{*} E\right)$ by $\left(f^{*} s\right)(\tilde{p}):=\left(s_{f}(\tilde{p}), \tilde{p}\right)$. If $f$ is a biholomorphic map, then there is a natural bundle isomorphism $f^{*}: E \rightarrow f^{*} E$ which covers $f^{-1}$. Finally, if $\nabla$ is a connection on $E$, then the pullback connection $f^{*} \nabla$ on $f^{*} E$ is defined by $\left(f^{*} \nabla\right)_{X_{\tilde{p}}}\left(f^{*} s\right):=f^{*}\left(\nabla_{f_{*}\left(X_{\tilde{p}}\right)}(s)\right)$, for any $X_{\tilde{p}} \in T_{\tilde{p}} \tilde{N}, \tilde{p} \in \tilde{N}$ and $s \in \mathcal{O}(E)$. 
Notation 4 For simplicity, we will say that a $(T)$ or $(T E)$-structure isomorphism as in Definition 3 covers $h$ instead of Id $\times h$ and write $h^{*}$ for the pullback (Id $\left.\times h\right)^{*}$ (of bundles, connections, etc.). Similarly, we will sometimes write $A_{i} \circ h$ instead of $A_{i} \circ(\operatorname{Id} \times h)$ and $T \circ h$ instead of $T \circ(\mathrm{Id} \times h)$.

The next lemma can be checked directly.

Lemma 5 Let $(\tilde{H}, \tilde{\nabla})$ and $(H, \nabla)$ be two $(T)$-structures over $\tilde{M}$ and $M$, respectively. If $T:(\tilde{H}, \tilde{\nabla}) \rightarrow(H, \nabla)$ is an isomorphism which covers $h: \tilde{M} \rightarrow M$, then $h^{*} \circ T:$ $(\tilde{H}, \tilde{\nabla}) \rightarrow\left(h^{*} H, h^{*} \nabla\right)$ is an isomorphism which covers the identity map of $\tilde{M}$.

Let $T:(\tilde{H}, \tilde{\nabla}) \rightarrow(H, \nabla)$ be a $(T)$ or $(T E)$-structure isomorphism over $\tilde{M}$ and $M$, respectively, which covers a biholomorphic map $h: \tilde{M} \rightarrow M$. Fix trivializations $\underline{\tilde{s}}=$ $\left(\tilde{s}_{1}, \ldots, \tilde{s}_{r}\right)$ and $\underline{s}=\left(s_{1}, \ldots, s_{r}\right)$ of $\tilde{H}$ and $H$ over $\Delta \times \tilde{U}$ and $\Delta \times U$, respectively, where $\tilde{U} \subset \tilde{M}, U \subset \bar{M}$ are open subsets and $U=h(\tilde{U})$. Then, the isomorphism $T$ is given by a matrix $\left(T_{i j}\right)=\sum_{k>0} T(k) z^{k} \in M\left(r \times r, \mathcal{O}_{\Delta \times U}\right)$ with $T(k) \in M\left(r \times r, \mathcal{O}_{U}\right), T(0)$ invertible, such that $T\left(\tilde{s}_{i}\right)=\sum_{j=1}^{r} T_{j i} s_{j}$, or, explicitly,

$$
T\left(\left(\tilde{s}_{i}\right)_{(z, \tilde{p})}\right)=\sum_{j=1}^{r} T_{j i}(z, h(\tilde{p}))\left(s_{j}\right)_{(z, h(\tilde{p}))}, \quad \forall \tilde{p} \in \tilde{U}, \quad z \in \Delta .
$$

We write relation (11) shortly as

$$
T\left((\underline{\tilde{s}})_{(z, \tilde{p})}\right)=(\underline{s})_{(z, h(\tilde{p}))} \cdot T(z, h(\tilde{p})) .
$$

Suppose now that $\left(\tilde{t}_{1}, \ldots, \tilde{t}_{m}\right)$ and $\left(t_{1}, \ldots, t_{m}\right)$ are local coordinates of $\tilde{M}$ and $M$, defined on $\tilde{U}$ and $U$, respectively. The compatibility relations (9), (10) read

$$
\begin{gathered}
z \partial_{i} \tilde{T}+\sum_{j=1}^{m}\left(\partial_{i} h^{j}\right)\left(A_{j} \circ h\right) \tilde{T}-\tilde{T} \tilde{A}_{i}=0, \quad \forall i \\
z^{2} \partial_{z} \tilde{T}+(B \circ h) \tilde{T}-\tilde{T} \tilde{B}=0,
\end{gathered}
$$

where $\tilde{T}:=T \circ h$ and $\left(h^{j}\right)$ are the components of the representation of $h$ in the two charts [relation (13) has to be omitted when $\tilde{\nabla}$ and $\nabla$ are (T)-structures]. Relations (12), (13) split according to the powers of $z$ as

$$
\begin{gathered}
\partial_{i} \tilde{T}(k-1)+\sum_{l=0}^{k}\left(\sum_{j=1}^{m}\left(\partial_{i} h^{j}\right)\left(A_{j}(l) \circ h\right) \tilde{T}(k-l)-\tilde{T}(k-l) \tilde{A}_{i}(l)\right)=0 \\
(k-1) \tilde{T}(k-1)+\sum_{l=0}^{k}((B(l) \circ h) \tilde{T}(k-l)-\tilde{T}(k-l) \tilde{B}(l))=0
\end{gathered}
$$

for any $k \geq 0$, where $\tilde{T}(-1)=0$.

We now discuss a particular class of $(T)$ and $(T E)$-structure isomorphisms, called gauge isomorphisms. Consider a $(T)$ or a $(T E)$-structure $(H, \nabla)$ with $H$ trivial, and $\underline{s}=\left(s_{1}, \ldots, s_{r}\right)$ a trivialization of $H$. Any other trivialization $\underline{\tilde{s}}=\left(\tilde{s}_{1}, \ldots, \tilde{s}_{r}\right)$ of $H$ is related to $\underline{s}$ by an invertible holomorphic matrix-valued function $T=\left(T_{i j}\right)$ defined by $\tilde{s}_{i}=\sum_{j=1}^{r} T_{j i} s_{j}$ (short: $\underline{\tilde{s}}=\underline{s} \cdot T$ ). Suppose that the connection form $\Omega$ of $\nabla$ in the trivialization $\underline{s}$ is given by (3), (4) [without the term $B$, when $\nabla$ is a (T)-structure]. Then, the 
connection form $\tilde{\Omega}$ of $\nabla$ in the new trivialization $\underline{\tilde{s}}$ has the same form, with matrices $\tilde{A}_{i}$ and $\tilde{B}$ related to $A_{i}$ and $B$ by

$$
\begin{aligned}
& z \partial_{i}(T)+A_{i} T-T \tilde{A}_{i}=0 \\
& z^{2} \partial_{z}(T)+B T-T \tilde{B}=0,
\end{aligned}
$$

or by

$$
\begin{gathered}
\partial_{i} T(k-1)+\sum_{l=0}^{k}\left(A_{i}(l) T(k-l)-T(k-l) \tilde{A}_{i}(l)\right)=0, \\
(k-1) T(k-1)+\sum_{l=0}^{k}(B(l) T(k-l)-T(k-l) \tilde{B}(l))=0 .
\end{gathered}
$$

for any $k \geq 0$. We say that $T$ defines a gauge isomorphism between the $(T)$ (or $(T E)$ structures) with connection forms $\Omega$ and $\tilde{\Omega}$.

Remark 6 (i) Gauge isomorphisms are isomorphisms between $(T)$ or $(T E)$-structures over the same base $M$ which lift the identity map of $M$. Remark that relations (14), (15) with $h=1$ reduce to (18), (19).

(ii) $(T)$ and ( $T E$ )-structures can be defined also over germs of manifolds. In this case, we always assume that their underlying bundles are trivial. Isomorphisms between them which lift a given biholomorphic map of their parameter spaces are given simply by matrices, as explained above (the matrix $T \in M\left(r \times r, \mathcal{O}_{\Delta \times U}\right)$ in the above notation).

(iii) $(T)$ and (TE)-structures can be extended to the formal setting as follows. A formal $(T)$ or $(T E)$-structure over a germ $(M, 0)$ is a pair $(H, \nabla)$, where $H \rightarrow(\mathbb{C}, 0) \times(M, 0)$ is the germ of a holomorphic vector bundle and $\nabla$ is given by a connection form (3), where $A_{i}$ and $B$ [the latter only when $\nabla$ is a $(T E)$-structure] are matrices with entries in $\mathbb{C}\{t, z]]$, satisfying relations (5), (6) or (7), (8) (relations (6), (8) only when $\nabla$ is a $(T E)$-structure). A formal isomorphism between two formal $(T)$ or $(T E)$-structures $(H, \nabla)$ and $(\tilde{H}, \tilde{\nabla})$ over $(M, 0)$ and $(\tilde{M}, 0)$, respectively, which covers a biholomorphic map $h:(\tilde{M}, 0) \rightarrow(M, 0)$, is given by a matrix $T=\left(T_{i j}\right)$ with entries $\left.T_{i j} \in \mathbb{C}\{t, z]\right]$, such that relations (12), (13) or (14), (15) are satisfied with $\tilde{T}=T \circ h$ (relations (13), (15) only when $\nabla$ and $\tilde{\nabla}$ are (TE)-structures). Formal gauge isomorphisms between $(T)$ or $(T E)$-structures over the same germ $(M, 0)$ are formal isomorphisms which cover the identity map of $(M, 0)$. They are given by matrices $T=\left(T_{i j}\right)$ with entries in $\mathbb{C}\{t, z]]$ such that relations (16), (17) or (18), (19) are satisfied.

\section{$2.2(T)$-structures and $F$-manifolds}

\subsubsection{General results}

Let $(H, \nabla)$ be a $(T)$-structure over a manifold $M$. It induces a vector valued 1 -form $C \in$ $\Omega^{1}(M, \operatorname{End}(K))$ on the restriction $K:=H_{\mid\{0\} \times M}$, defined by

$$
C_{X}[a]:=\left[z \nabla_{X} a\right], \quad \forall X \in \mathcal{T}_{M}, a \in \mathcal{O}(H),
$$

where [ ] means the restriction to $\{0\} \times M$ and $X \in \mathcal{T}_{M}$ is lifted canonically from its domain of definition $U \subset M$ to $\mathbb{C} \times U$. In the notation from Sect. 2.1, $C$ is given locally by $\sum_{i=1}^{m} A_{i}(0) \mathrm{d} t_{i}$. Relation (7) with $k=0$ implies $\left[A_{i}(0), A_{j}(0)\right]=0$, i.e., $C_{X} C_{Y}=C_{Y} C_{X}$ 
for any $X, Y \in T M$, which we write as $C \wedge C=0$. We say that $C$ is a $\operatorname{Higgs~field~and~}(K, C)$ is a Higgs bundle.

If $(H, \nabla)$ is a $(T E)$-structure, then there is in addition an endomorphism $\mathcal{U} \in \operatorname{End}(K)$,

$$
\mathcal{U}:=\left[z \nabla_{z} \partial_{z}\right]: \mathcal{O}(K) \rightarrow \mathcal{O}(K) .
$$

It satisfies $C_{X} \mathcal{U}=\mathcal{U} C_{X}$ for any $X \in T M$. We write this as $[C, \mathcal{U}]=0$.

Definition 7 [11, Theorem 2.5]

(a) A Higgs bundle $(K \rightarrow M, C)$ satisfies the unfolding condition if there is an open cover $\mathcal{V}$ of $M$ such that, on any $U \in \mathcal{V}$, there is $\zeta \in \mathcal{O}\left(\left.K\right|_{U}\right)$ (called a primitive section) with the property that the map $\left.T U \ni X \rightarrow C_{X} \zeta \in K\right|_{U}$ is an isomorphism.

(b) A $(T)$-structure [or a (TE)-structure] satisfies the unfolding condition if the induced Higgs bundle satisfies the unfolding condition.

Remark 8 If $(H \rightarrow \mathbb{C} \times M, \nabla)$ is a $(T)$-structure with the unfolding condition, then the rank of $H$ and the dimension of $M$ coincide.

We now define the parallel notion of $F$-manifold.

Definition 9 [10] A complex manifold $M$ with a fiber-preserving, commutative, associative multiplication $\circ$ on the holomorphic tangent bundle $T M$ and unit field $e \in \mathcal{T}_{M}$ is an $F$ manifold if

$$
L_{X \circ Y}(\circ)=X \circ L_{Y}(\circ)+Y \circ L_{X}(\circ), \quad \forall X, Y \in \mathcal{T}_{M} .
$$

A vector field $E \in \mathcal{T}_{M}$ is called an Euler field (of weight 1) if

$$
L_{E}(\text { ○) }=\text { o. }
$$

The following lemma was proved in Theorem 3.3 of [13]. The proof below is more elegant and shorter.

Lemma $10 A(T)$-structure $(H \rightarrow \mathbb{C} \times M, \nabla)$ with unfolding condition induces a multiplication $\circ$ on $T M$ which makes $M$ an $F$-manifold. $A(T E)$-structure $(H \rightarrow \mathbb{C} \times M, \nabla)$ with unfolding condition induces in addition a vector field $E$ on $M$, which, together with $\circ$, makes $M$ an $F$-manifold with Euler field.

Proof Let $(H \rightarrow \mathbb{C} \times M, \nabla)$ be a $(T)$ or $(T E)$-structure with induced Higgs bundle $(K, C)$. We define $\circ$ by

$$
C_{X \circ Y} \zeta=C_{X} C_{Y} \zeta, \quad \forall X, Y \in T M,
$$

where $\zeta$ is a local primitive section. We remark that $\circ$ has unit field $e$ determined by the condition $C_{e} \zeta=\zeta$. When $\nabla$ is a $(T E)$-structure, the induced endomorphism $\mathcal{U}$ of $K$ defines a unique vector field $E \in \mathcal{T}_{M}$ with $-C_{E} \zeta=\mathcal{U}(\zeta)$. The definition of $\circ$ and $E$ are independent on the choice of $\zeta$ (see Lemma 4.1 of [8]).

Suppose now that $\nabla$ is a $(T)$-structure. In order to prove that $(M, \circ, e)$ is an $F$-manifold, it is sufficient to find a $(1,0)$-connection $D^{\prime}$ on the (complex) $C^{\infty}$-bundle underlying $T M$, with

$$
D^{\prime}\left(C^{\prime}\right)_{X, Y}:=D_{X}^{\prime}\left(C_{Y}^{\prime}\right)-D_{Y}^{\prime}\left(C_{X}^{\prime}\right)-C_{[X, Y]}^{\prime}=0, \quad \forall X, Y \in \mathcal{T}_{M}
$$

where $C_{X}^{\prime} Y:=X \circ Y$. The sufficiency follows with Lemma 4.3 of [8].

If $\nabla$ is a $(T E)$-structure, in order to prove that $(M, \circ, e, E)$ is an $F$-manifold with Euler field it is sufficient to prove in addition the existence of a $C^{\infty}$-endomorphism $\mathcal{Q}^{\prime}$ of $T M$, with

$$
D_{X}^{\prime}\left(\mathcal{U}^{\prime}\right)-\left[C_{X}^{\prime}, \mathcal{Q}^{\prime}\right]+C_{X}^{\prime}=0, \quad \forall X \in \mathcal{T}_{M},
$$


where $\mathcal{U}^{\prime}(X):=-E \circ X$ for any $X \in T M$. The sufficieny follows again from Lemma 4.3 of [8].

We will define $D^{\prime}$ and $\mathcal{Q}^{\prime}$ locally, on any open subset $U \subset M$, small enough such that there is a primitive section $\zeta$ of $\left.K\right|_{U}$ and a coordinate system $\left(t_{1}, \ldots, t_{m}\right)$ of $M$ defined on $U$. Let $\partial_{1}, \ldots, \partial_{m}$ be the associated coordinate vector fields. Let $\underline{s}:=\left(s_{1}, \ldots, s_{m}\right)$ be a trivialization of $H$ on $\Delta \times U$ (where $\Delta$ is a small disk centered at $0 \in \mathbb{C}$ ) and let $\Omega=\frac{1}{z} \sum_{i=1}^{m} A_{i} \mathrm{~d} t_{i}+\frac{1}{z^{2}} B \mathrm{~d} z$ be the connection form of $\nabla$ in this trivialization [with $B=0$ when $\nabla$ is a $(T)$-structure]. Let $\left.\underline{s}\right|_{\{0\} \times U}=: \underline{s}^{(0)}=\left(s_{1}^{(0)}, \ldots, s_{m}^{(0)}\right)$ be the trivialization of $K$ obtained by restricting $\underline{s}$ to $\{0\} \times U$. Define a $(1,0)$-connection $D$ on $\left.K\right|_{U}$, by

$$
D_{\partial_{k}}\left(s_{i}^{(0)}\right)=\sum_{j=1}^{m} A_{k}(1)_{j i} s_{j}^{(0)}, \quad \text { short: } D_{\partial_{k}}\left(\underline{s}^{(0)}\right)=\underline{s}^{(0)} \cdot A_{k}(1),
$$

and, when $\nabla$ is a $(T E)$-structure, an endomorphism $\mathcal{Q}$ of $\left.K\right|_{U}$ by

$$
\mathcal{Q}\left(s_{i}^{(0)}\right):=-\sum_{j=1}^{m} B(1)_{j i} s_{j}^{(0)} \text {. short: } \mathcal{Q}\left(\underline{s}^{(0)}\right)=-\underline{s}^{(0)} \cdot B(1) .
$$

From relations (5) and (6),

$$
\begin{gathered}
\partial_{j} A_{k}(0)-\partial_{k} A_{j}(0)+\left[A_{j}(1), A_{k}(0)\right]+\left[A_{j}(0), A_{k}(1)\right]=0 \\
\partial_{j} B(0)+A_{j}(0)+\left[A_{j}(1), B(0)\right]+\left[A_{j}(0), B(1)\right]=0 .
\end{gathered}
$$

From the definitions of $C$ and $\mathcal{U}$,

$$
\begin{aligned}
C_{\partial_{j}}\left(s_{r}^{(0)}\right) & =\sum_{k=1}^{m} A_{j}(0)_{k r} s_{k}^{(0)}, \quad \text { short: } C_{\partial_{j}}\left(\underline{s}^{(0)}\right)=\underline{s}^{(0)} \cdot A_{j}(0), \\
\mathcal{U}\left(s_{r}^{(0)}\right) & =\sum_{k=1}^{m} B(0)_{k r} s_{k}^{(0)}, \quad \operatorname{short}: \mathcal{U}\left(\underline{s}^{(0)}\right)=\underline{s}^{(0)} \cdot B(0) .
\end{aligned}
$$

Now, a straightforward computation shows that (29) gives

$$
D(C)_{X, Y}=0, \quad \forall X, Y \in \mathcal{T}_{M}
$$

and (30) gives

$$
D_{X}(\mathcal{U})-\left[C_{X}, \mathcal{Q}\right]+C_{X}=0, \quad \forall X \in \mathcal{T}_{M} .
$$

More precisely, (33) is obtained from the following computation:

$$
\begin{aligned}
D(C)_{\partial_{j}, \partial_{r}}\left(\underline{s}^{(0)}\right)= & \left(D_{\partial_{j}}\left(C_{\partial r}\right)-D_{\partial_{r}}\left(C_{\partial j}\right)\right)\left(\underline{s}^{(0)}\right) \\
= & D_{\partial_{j}}\left(C_{\partial r}\left(\underline{s}^{(0)}\right)\right)-C_{\partial_{r}}\left(D_{\partial_{j}}\left(\underline{s}^{(0)}\right)\right)-D_{\partial_{r}}\left(C_{\partial j}\left(\underline{s}^{(0)}\right)\right)+C_{\partial_{j}}\left(D_{\partial_{r}}\left(\underline{s}^{(0)}\right)\right) \\
= & \underline{s}^{(0)} \cdot\left[\partial_{j}\left(A_{r}(0)\right)+A_{j}(1) A_{r}(0)-A_{r}(0) A_{j}(1)\right. \\
& \left.-\partial_{r}\left(A_{j}(0)\right)-A_{r}(1) A_{j}(0)+A_{j}(0) A_{r}(1)\right] \\
= & \underline{s}^{(0)} \cdot\left[\partial_{j} A_{r}(0)-\partial_{r} A_{j}(0)+\left[A_{j}(1), A_{r}(0)\right]+\left[A_{j}(0), A_{r}(1)\right]\right] .
\end{aligned}
$$

which vanishes from (29). Relation (34) can be proved similarly.

By means of the isomorphism $\left.T U \cong K\right|_{U}$ defined by $X \rightarrow C_{X} \zeta$, where $\zeta$ is a primitive section on $U$, the connection $D^{\prime}$ and endomorphism $\mathcal{Q}^{\prime}$ we are looking for (the latter when $\nabla$ is a $(T E)$-structure) correspond to the connection $D$ and endomorphism $\mathcal{Q}$, respectively. 
When $\nabla$ is a $(T E)$-structure, the endomorphisms $\mathcal{U}$ and $\mathcal{U}^{\prime}$ also correspond. Relations (25) and (26) follow from (33) and (34), respectively.

Let $h:(\tilde{M}, \tilde{o}, \tilde{e}) \rightarrow(M, \circ, e)$ be an $F$-manifold isomorphism. If $(E, \nabla)$ is a $(T)$-structure over $M$ which induces $(\circ, e)$, then $\left(h^{*} E, h^{*} \nabla\right)$ is a $(T)$-structure over $\tilde{M}$ which induces ( $(\tilde{\circ}, \tilde{e})$ (and a similar statement holds for ( $T E$ )-structures and $F$-manifolds with Euler fields). In particular, the spaces of $(T)$-structures over isomorphic $F$-manifolds (or isomorphic germs of $F$-manifolds) are isomorphic. The same statement is true for the spaces of formal $(T)$ structures over isomorphic germs of $F$-manifolds. (The unfolding condition can be extended, in the obvious way, to formal $(T)$ and $(T E)$-structures, and Lemma 10 remains true when the $(T)$ or $(T E)$-structure is replaced by a formal one.)

\subsubsection{Germs of two-dimensional $F$-manifolds}

There exist two types of isomorphism classes of irreducible germs of two-dimensional $F$ manifolds (see [9], Theorem 4.7): $I_{2}(m)$ with $m \in \mathbb{N}_{\geq 3}$ (generically semisimple) and $\mathcal{N}_{2}$ (globally nilpotent). As germs of manifolds, $I_{2}(m)$ and $\mathcal{N}_{2}$ are $\left(\mathbb{C}^{2}, 0\right)$. In the standard coordinates $\left(t_{1}, t_{2}\right)$ of $\mathbb{C}^{2}$, the multiplication of $I_{2}(m)$ has $\partial_{1}$ as unit field and $\partial_{2} \circ \partial_{2}=t_{2}^{m-2} \partial_{1}$. Similarly, the multiplication of $\mathcal{N}_{2}$ has $\partial_{1}$ as unit field and $\partial_{2} \circ \partial_{2}=0$. The next simple lemma describes the automorphism groups of $I_{2}(m)$ and $\mathcal{N}_{2}$.

Lemma 11 (i) The automorphism group of $I_{2}(m)(m \geq 3)$ is cyclic of order $m$, generated by the automorphism

$$
\left(t_{1}, t_{2}\right) \rightarrow\left(t_{1}, e^{\frac{2 \pi i}{m}} t_{2}\right) .
$$

(ii) The automorphism group of $\mathcal{N}_{2}$ is the group of all biholomorphic maps

$$
\left(t_{1}, t_{2}\right) \rightarrow\left(t_{1}, \lambda\left(t_{2}\right)\right)
$$

where $\lambda \in \mathbb{C}\left\{t_{2}\right\}$, with $\lambda(0)=0$ and $\dot{\lambda}(0) \neq 0$.

Our aim in this paper is to find normal forms, up to formal isomorphisms, for $(T)$ structures over an arbitrary irreducible germ $(M, 0)$ of two-dimensional $F$-manifolds. From the comments which end Sect. 2.2.1, we can (and will) assume, without loss of generality, that $(M, 0)$ is either $I_{2}(m)(m \geq 3)$ or $\mathcal{N}_{2}$.

\section{Differential equations}

We now prove various results on differential equations which will be useful in the next sections. Along this section $t \in(\mathbb{C}, 0)$ is the standard coordinate.

Lemma 12 Consider the differential equation

$$
\frac{\mathrm{d}}{\mathrm{d} t}(a h)+a \frac{\mathrm{d} h}{\mathrm{~d} t}=c,
$$

where $a, c \in \mathbb{C}\{t\}$ are given and the function $h=h(t)$ is unknown.

(i) If $a(0) \neq 0$, then there is a unique formal solution $h$ with given $h(0) \in \mathbb{C}$ and this solution is holomorphic. 
(ii) If $t=0$ is a zero of order one for $a$, then there is a unique formal solution of (36) and this solution is holomorphic.

(iii) If $t=0$ is a zero of order $o \geq 2$ for a, then (36) has a formal solution if and only if $t=0$ is a zero of order at least $o-1$ for $c$. When it exists, the formal solution is unique and holomorphic.

In all cases, if $a$ and c converge on $\Delta$ (an open disk centered at $0 \in \mathbb{C}$ ), then also the formal solution converges on $\Delta$.

Proof As the proof is elementary, we skip the details. Claim (i) follows from the fundamental theorem of differential equations. For claims (ii) and (iii), one checks easily (by taking power series and identifying coefficients) the part concerning the existence of formal solutions. For the convergence, one uses the general result that any formal solution $u(t)=\sum_{n \geq 0} u_{n} t^{n}$ of a differential equation of the form

$$
t \dot{u}(t)+A(t) u(t)=b(t),
$$

where $A: \Delta \rightarrow M_{n}(\mathbb{C})$ and $b: \Delta \rightarrow \mathbb{C}^{n}$ are holomorphic, is convergent on $\Delta$. This was proved, e.g., in Theorem 5.3 of [20] (see page 22), when $b=0$. The case $b \neq 0$ can be reduced to the case $b=0$ in the standard way: if $u=\left(u_{1}, \ldots, u_{n}\right)^{t}$ is a solution of (37) with $b \neq 0$, one defines $v:=\left(u_{1}, \ldots, u_{n}, 1\right)^{t}$ and sees that $v$ satisfies a differential equation (in dimension $n+1$ ) of the same type (37) but with $b=0$. One easily shows that in claims (ii) and (iii) equation (36) reduces to an equation of the form (37) (with $A$ and $b$ scalar functions). We obtain that the formal solution of (36), in these cases, converges on $\Delta$ if $a$ and $c$ do.

For a given function $f:(\mathbb{C}, 0) \rightarrow(\mathbb{C}, 0)$ and $n \in \mathbb{Z}_{\geq 1}$ we denote by $f^{n}$ the function $f^{n}(t):=f(t) \cdot \cdots \cdot f(t)$ (multiplication $n$-times; not to be confused with the iterated composition $f \circ \cdots \circ f)$.

Lemma 13 Let $f \in \mathbb{C}\{t\}$ be non-trivial and $r:=\operatorname{ord}_{0}(f)$. Then there is $\lambda \in \mathbb{C}\{t\}$, with $\lambda(0)=0$ and $\dot{\lambda}(0) \neq 0$, such that $(\dot{\lambda})^{2} \lambda^{r}=f$. Moreover, any two such functions $\lambda$ and $\tilde{\lambda}$ are related by $\tilde{\lambda}(t)=\lambda_{0} \lambda(t)$, where $\lambda_{0} \in \mathbb{C}, \lambda_{0}^{r+2}=1$.

Proof As $r=\operatorname{ord}_{0}(f)$, we can write $f(t)=t^{r} g(t)$ with $g \in \mathbb{C}\{t\}$ a unit. Similarly, the function $\lambda$ we are looking for is of the form $\lambda(t)=t x(t)$, with $x \in \mathbb{C}\{t\}$ a unit. We are looking for $x$ which satisfies the differential equation

$$
(x+t \dot{x})^{2} x^{r}=g .
$$

As $g(0) \neq 0$, there is $k \in \mathbb{C}\{t\}$ a unit, such that $g=k^{2}$. Similarly, as $x(0) \neq 0$ we can write $x=z^{2}$, for $z \in \mathbb{C}\{z\}$. Equation (38) is satisfied if $(x+t \dot{x}) z^{r}=k$ or

$$
2 t\left(z^{r+2}\right)^{\prime}+(r+2) z^{r+2}=(r+2) k .
$$

The differential equation in the unknown function $y$

$$
2 t \dot{y}+(r+2) y=(r+2) k
$$

has a unique formal solution. From Lemma 12, this solution is holomorphic. As $k(0) \neq 0$, we obtain $y(0) \neq 0$. Let $z \in \mathbb{C}\{t\}$ such that $z^{r+2}=y$. The function $z$ satisfies (39) and $\lambda(t):=t z(t)^{2}$ satisfies $(\dot{\lambda})^{2} \lambda^{r}=f$, as needed. The first statement is proved. The second statement follows by taking into account the freedom in the choice of $z$ and $k$ in the above argument. 
Lemma 14 Consider the system of two differential equations

$$
\begin{gathered}
\dot{g}_{1}+g_{2} h_{1}+h_{2}=0, \\
\left(r+t \frac{\mathrm{d}}{\mathrm{d} t}\right)\left(g_{2}\right)+g_{1} h_{3}+h_{4}=0,
\end{gathered}
$$

where $r \in \mathbb{R}_{>0}, h_{1}, h_{2}, h_{3}, h_{4} \in \mathbb{C}\{t\}$ are given, and the functions $g_{1}=g_{1}(t)$ and $g_{2}=g_{2}(t)$ are unknown.

For any choice of $g_{1}(0) \in \mathbb{C}$, there is a unique formal solution $\left(g_{1}, g_{2}\right)$ of (40), (41), and this is holomorphic. If $h_{1}, h_{2}, h_{3}$ and $h_{4}$ converge on $\Delta$ (an open disk centered at $0 \in \mathbb{C}$ ), then also $g_{1}$ and $g_{2}$ converge on $\Delta$.

Proof Writing $g_{i}=\sum_{k \geq 0} g_{i}^{(k)} t^{k}$ and $h_{j}=\sum_{k \geq 0} h_{j}^{(k)} t^{k}$, the two differential equations are equivalent to the following equations,

$$
\begin{aligned}
& n g_{1}^{(n)}+\sum_{k=0}^{n-1} g_{2}^{(k)} h_{1}^{(n-1-k)}+h_{2}^{(n-1)}=0, \quad \forall n \in \mathbb{Z}_{\geq 1}, \\
& (r+n) g_{2}^{(n)}+\sum_{k=0}^{n} g_{1}^{(k)} h_{3}^{(n-k)}+h_{4}^{(n)}=0, \quad \forall n \in \mathbb{Z}_{\geq 0} .
\end{aligned}
$$

Let $g_{1}^{(0)} \in \mathbb{C}$ be given. Then, Eqs. (42), (43) determine inductively all coefficients $g_{1}^{(n)}$, for $n \geq 1$, and $g_{2}^{(n)}$, for $n \geq 0$. We obtain a unique formal solution $\left(g_{1}, g_{2}\right)$. From the proof of Lemma 12, $g_{1}$ and $g_{2}$ are holomorphic. [Multiplying (40) by $t$, we notice that (40), (41) is of the form (37).]

\section{$4(T)$-structures over $I_{2}(m)$}

In this section, we find formal normal forms for $(T)$-structures over the germ $I_{2}(m)(m \geq 3)$. We need to introduce notation.

Notation 15 Along this section, $\left(t_{1}, t_{2}\right)$ denote the standard coordinates on $\mathbb{C}^{2}$. We shall use the following matrices

$$
C_{1}:=\operatorname{Id}_{2}, \quad C_{2}:=\left(\begin{array}{cc}
0 & t_{2}^{m-2} \\
1 & 0
\end{array}\right), \quad D:=\left(\begin{array}{cc}
1 & 0 \\
0 & -1
\end{array}\right), \quad E:=\left(\begin{array}{ll}
0 & 1 \\
0 & 0
\end{array}\right),
$$

and the relations between them:

$$
\begin{aligned}
& \left(C_{2}\right)^{2}=t_{2}^{m-2} C_{1}, \quad D^{2}=C_{1}, \quad E^{2}=0, \\
& C_{2} D=C_{2}-2 t_{2}^{m-2} E=-D C_{2}, \\
& C_{2} E=\frac{1}{2}\left(C_{1}-D\right), \quad E C_{2}=\frac{1}{2}\left(C_{1}+D\right), \\
& D E=E=-E D .
\end{aligned}
$$

Remark that

$$
\left[C_{2}, D\right]=2\left(C_{2}-2 t_{2}^{m-2} E\right), \quad\left[C_{2}, E\right]=-D, \quad[D, E]=2 E .
$$

The matrices $C_{1}, C_{2}, D$ and $E$ form an $\mathcal{O}_{\left(\mathbb{C}^{2}, 0\right)}$-basis of $M\left(2 \times 2, \mathcal{O}_{\left(\mathbb{C}^{2}, 0\right)}\right)$. 
Theorem 16 (i) produces a non-unique normal form, with respect to gauge isomorphisms, for any $(T)$-structure over $I_{2}(m)(m \geq 3)$. Theorem 16 (iii) produces a unique normal form, with respect to formal gauge isomorphisms, for any formal or holomorphic $(T)$-structure over such a germ.

Theorem 16 (i) Over the germ $I_{2}(m)(m \geq 3)$, any (T)-structure is gauge isomorphic to a (T)-structure of the form

$$
A_{1}=C_{1}, \quad A_{2}=C_{2}+z f E,
$$

where $f \in \mathbb{C}\{t, z\}$ is holomorphic.

(ii) Any formal $(T)$-structure over $I_{2}(m)$ is formally gauge isomorphic to a formal $(T)$ structure of the form (47) where $f \in \mathbb{C}\{t, z]]$.

(iii) Any holomorphic or formal $(T)$-structure over $I_{2}(m)$ is formally gauge isomorphic to a formal (T)-structure of the form (47) where $f=0$ if $m=3$ and $f \in \mathbb{C}[[z]]\left[t_{2}\right]_{\leq m-4}$ if $m \geq 4$. With respect to formal gauge isomorphisms, the function $f$ is unique.

Proof To prove claim (i), we start with an arbitrary $(T)$-structure $(H, \nabla)$ over $I_{2}(m)$ with $m \geq 3$. We choose a trivialization $\underline{s}=\left(s_{1}, s_{2}\right)$ of $H$ such that the connection form $\Omega$ of $\nabla$ is given by $\Omega=\frac{1}{z}\left(A_{1} \mathrm{~d} t_{1}+A_{2} \mathrm{~d} t_{2}\right)$, where

$$
A_{1}(0)=C_{1}, \quad A_{2}(0)=C_{2} .
$$

We will reduce $\nabla$ to the required (non-unique) normal form in three steps.

The first step of the normalization is the reduction of $A_{1}$ to $C_{1}$ and of $A_{2}$ to a new matrix $\widetilde{A}_{2}$ with $\widetilde{A}_{2}(0)=C_{2}$ and $\partial_{1} \widetilde{A}_{2}=0$. Consider the system

$$
\partial_{1} T=-\left(\sum_{k \geq 1} A_{1}(k) z^{k-1}\right) T, \quad T\left(z, 0, t_{2}\right)=C_{1} .
$$

It has a unique holomorphic solution $T$. We write $T=\sum_{k \geq 0} T(k) z^{k}$ with $T(k)$ independent of $z$. We claim that

$$
T(0) \in \mathcal{O}_{\left(\mathbb{C}^{2}, 0\right)} \cdot C_{1}+\mathcal{O}_{\left(\mathbb{C}^{2}, 0\right)} \cdot C_{2} .
$$

To prove this claim, we remark that (49) for $z=0$ gives

$$
\partial_{1} T(0)=-A_{1}(1) T(0), \quad T(0)\left(0, t_{2}\right)=C_{1} .
$$

On the other hand, relation (7) for $k=1$ together with (48) gives

$$
0=\partial_{1} A_{2}(0)-\partial_{2} A_{1}(0)+\left[A_{1}(0), A_{2}(1)\right]+\left[A_{1}(1), A_{2}(0)\right]=\left[A_{1}(1), C_{2}\right],
$$

which implies $A_{1}(1)=a_{1} C_{1}+a_{2} C_{2}$ for $a_{1}, a_{2} \in \mathcal{O}_{\left(\mathbb{C}^{2}, 0\right)}$. The differential equation (51) with $A_{1}(1)$ of this form and ansatz $T(0)=\tau_{01} C_{1}+\tau_{02} C_{2}$, with $\tau_{01}, \tau_{02} \in \mathcal{O}_{\left(\mathbb{C}^{2}, 0\right)}$, and $\tau_{01}\left(0, t_{2}\right)=1, \tau_{02}\left(0, t_{2}\right)=0$, has a unique solution. We obtain that $T$ satisfies $(50)$, as required. We now change the trivialization $\underline{s}$ by means of $T$. In the new trivialization, $\nabla$ is given by matrices $\widetilde{A}_{1}$ and $\widetilde{A}_{2}$. From (12) for $i=1$, together with $A_{1}(0)=C_{1}$ and (49), we obtain:

$$
0=z \partial_{1} T+A_{1} T-T \widetilde{A}_{1}=C_{1} T-T \widetilde{A}_{1}=T\left(C_{1}-\widetilde{A}_{1}\right)
$$

which implies $\widetilde{A}_{1}=C_{1}$. From (18) for $k=0$ and $i=2$,

$$
0=A_{2}(0) T(0)-T(0) \widetilde{A}_{2}(0)=T(0)\left(C_{2}-\widetilde{A}_{2}(0)\right),
$$


where we used (50) and $A_{2}(0)=C_{2}$. We obtain $\widetilde{A}_{2}(0)=C_{2}$. Finally, from (16),

$$
0=z \partial_{1} \widetilde{A}_{2}-z \partial_{2} \widetilde{A}_{1}+\left[\widetilde{A}_{1}, \widetilde{A}_{2}\right]=z \partial_{1} \widetilde{A}_{2}
$$

from which we deduce $\partial_{1} \widetilde{A}_{2}=0$. The first step is completed.

Owing to the first step, from now on we assume that $A_{1}=C_{1}, A_{2}(0)=C_{2}$ and $\partial_{1} A_{2}=0$.

The second step does not change $A_{1}=C_{1}$ and erases the term $C_{1}$ in $A_{2}$. Suppose that

$$
A_{2}=C_{2}+z\left(a_{1} C_{1}+a_{2} C_{2}+a_{3} D+a_{4} E\right)
$$

with $a_{1}, a_{2}, a_{3}, a_{4} \in \mathbb{C}\left\{z, t_{2}\right\}$. Let $\tau_{1} \in \mathbb{C}\left\{z, t_{2}\right\}$ be the unique solution of

$$
\partial_{2} \tau_{1}=-a_{1} \tau_{1}, \quad \tau_{1}(z, 0)=1
$$

and $T:=\tau_{1} C_{1}$. Relation (16) for $i=2$ gives

$$
\left.0=z \partial_{2} T+A_{2} T-T \widetilde{A}_{2}=\left(C_{2}+z\left(a_{2} C_{2}+a_{3} D+a_{4} E\right)\right)-\widetilde{A}_{2}\right) T .
$$

Thus,

$$
\widetilde{A}_{2}=C_{2}+z\left(a_{2} C_{2}+a_{3} D+a_{4} E\right),
$$

as needed. Remark that the coefficients of $C_{2}, D$ and $E$ in the expressions (56) and (58) of $A_{2}$ and $\tilde{A}_{2}$ are same.

The third step of the reduction does not change $A_{1}=C_{1}$ and brings $A_{2}$ to the form $C_{2}+z f E$ with $f \in \mathbb{C}\left\{z, t_{2}\right\}$. Suppose

$$
A_{2}=C_{2}+z\left(a_{2} C_{2}+a_{3} D+a_{4} E\right)
$$

with $a_{2}, a_{3}, a_{4} \in \mathbb{C}\left\{z, t_{2}\right\}$. We are searching for $T$ and $\widetilde{A}_{2}$ of the form

$$
\begin{gathered}
T=C_{1}+z\left(\tau_{3} D+\tau_{4} E\right) \\
\widetilde{A}_{2}=C_{2}+z\left(\tilde{a}_{1} C_{1}+\tilde{a}_{4} E\right)
\end{gathered}
$$

where $\tau_{3}, \tau_{4}, \tilde{a}_{1}, \tilde{a}_{4} \in \mathbb{C}\left\{t_{2}, z\right\}$, which, together with $A_{2}$, satisfy (16) for $i=2$ :

$$
\begin{aligned}
0= & z \partial_{2} T+A_{2} T-T \widetilde{A}_{2} \\
= & z^{2} \partial_{2} \tau_{3} D+z^{2} \partial_{2} \tau_{4} E+z\left[C_{2}, \tau_{3} D+\tau_{4} E\right] \\
& +\left(C_{2}+z\left(a_{2} C_{2}+a_{3} D+a_{4} E\right)\right)-\left(C_{2}+z\left(\tilde{a}_{1} C_{1}+\tilde{a}_{4} E\right)\right) \\
& +z^{2}\left(a_{2} C_{2}+a_{3} D+a_{4} E\right)\left(\tau_{3} D+\tau_{4} E\right)-z^{2}\left(\tau_{3} D+\tau_{4} E\right)\left(\tilde{a}_{1} C_{1}+\tilde{a}_{4} E\right) \\
= & z^{2} \partial_{2} \tau_{3} D+z^{2} \partial_{2} \tau_{4} E+z\left(\tau_{3}\left(2 C_{2}-4 t_{2}^{m-2} E\right)-\tau_{4} D\right) \\
& +z\left(a_{2} C_{2}+a_{3} D+a_{4} E\right)-z\left(\tilde{a}_{1} C_{1}+\tilde{a}_{4} E\right) \\
& +z^{2}\left(a_{2} \tau_{3}\left(C_{2}-2 t_{2}^{m-2} E\right)+a_{3} \tau_{3} C_{1}-a_{4} \tau_{3} E\right. \\
& \left.+a_{2} \tau_{4} \frac{1}{2}\left(C_{1}-D\right)+a_{3} \tau_{4} E\right)-z^{2}\left(\tilde{a}_{1} \tau_{3} D+\tilde{a}_{1} \tau_{4} E_{4}+\tilde{a}_{4} \tau_{3} E\right) .
\end{aligned}
$$


Ordering the terms and dividing once by $z$, we obtain

$$
\begin{aligned}
0= & C_{1}\left(-\tilde{a}_{1}+z\left(a_{3} \tau_{3}+\frac{1}{2} a_{2} \tau_{4}\right)\right)+C_{2}\left(2 \tau_{3}+a_{2}+z a_{2} \tau_{3}\right) \\
& +D\left(-\tau_{4}+a_{3}+z\left(\partial_{2} \tau_{3}-\frac{1}{2} a_{2} \tau_{4}-\tilde{a}_{1} \tau_{3}\right)\right) \\
& +E\left(a_{4}-\tilde{a}_{4}-4 t_{2}^{m-2} \tau_{3}+z\left(\partial_{2} \tau_{4}-2 t_{2}^{m-2} a_{2} \tau_{3}-\left(a_{4}+\tilde{a}_{4}\right) \tau_{3}+a_{3} \tau_{4}-\tilde{a}_{1} \tau_{4}\right)\right) .
\end{aligned}
$$

The coefficient of $C_{2}$ determines $\tau_{3}$ uniquely $\left(2+z a_{2}\right.$ is a unit in $\left.\mathbb{C}\left\{z, t_{2}\right\}\right)$. The coefficient of $C_{1}$ determines $\tilde{a}_{1}$ in terms of $\tau_{4}$. The coefficient of $D$ determines then $\tau_{4}$. Finally, the coefficient of $E$ determines $\tilde{a}_{4}$. We proved that $A_{2}$ can be brought to the form (61). Applying the second step to $A_{1}=C_{1}$ and $\tilde{A}_{2}$ given by (61), we bring (without changing $\left.A_{1}=C_{1}\right) A_{2}$ to the form $C_{2}+z f E$ (with $f=\tilde{a}_{4}$ ), as needed. This completes the proof of claim (i).

The proof of claim (ii) is analogous, with series in $\left.\mathbb{C}\left\{t_{2}, z\right]\right]$ instead of functions in $\mathbb{C}\left\{t_{2}, z\right\}$.

Now we prove claim (iii). For this let two arbitrary formal normal forms $A_{1}, A_{2}$ and $\tilde{A_{1}}, \tilde{A_{2}}$, be given by

$$
A_{1}=\tilde{A_{1}}=C_{1}, \quad A_{2}=C_{2}+z f E, \quad \tilde{A}_{2}=C_{2}+z \tilde{f} E
$$

where $\left.f, \tilde{f} \in \mathbb{C}\left\{t_{2}, z\right]\right]$. We study when they are formally gauge isomorphic. This happens if there is a matrix-valued power series

$$
T=\tau_{1} C_{1}+\tau_{2} C_{2}+\tilde{\tau_{3}} D+\tilde{\tau_{4}} E
$$

with $\left.\tau_{1}, \tau_{2}, \tilde{\tau_{3}}, \tilde{\tau_{4}} \in \mathbb{C}\{t, z]\right]$, such that $T(0)(0)$ is invertible and

$$
z \partial_{j} T+A_{j} T-T \tilde{A_{j}}=0, \quad j \in\{1,2\} .
$$

Relation (66) for $j=1$ gives $\partial_{1} T=0$, or $\left.\tau_{1}, \tau_{2}, \tilde{\tau_{3}}, \tilde{\tau_{4}} \in \mathbb{C}\left\{t_{2}, z\right]\right]$. We write $\tau_{i}=$ $\sum_{n \geq 0} \tau_{i}(n) z^{n}$ with $\tau_{i}(n) \in \mathbb{C}\left\{t_{2}\right\}(i \in\{1,2\})$ and similarly $\tilde{\tau}_{i}=\sum_{n \geq 0} \tilde{\tau}_{i}(n) z^{n}$ with $\tilde{\tau}_{i}(n) \in \mathbb{C}\left\{t_{2}\right\}(i \in\{3,4\})$. Relation (66) for $j=2$ gives

$$
\begin{aligned}
0= & z \partial_{2} T+A_{2} T-T \widetilde{A}_{2} \\
= & z\left(\left(\partial_{2} \tau_{1}\right) C_{1}+\left(\partial_{2} \tau_{2}\right) C_{2}+(m-2) t_{2}^{m-3} \tau_{2} E+\left(\partial_{2} \tilde{\tau_{3}}\right) D+\left(\partial_{2} \tilde{\tau}_{4}\right) E\right) \\
& +\left[C_{2}, T\right]+z(f E T-\tilde{f} T E) \\
= & z\left(\left(\partial_{2} \tau_{1}\right) C_{1}+\left(\partial_{2} \tau_{2}\right) C_{2}+\left(\partial_{2} \tilde{\tau}_{3}\right) D+\left((m-2) t_{2}^{m-3} \tau_{2}+\partial_{2} \tilde{\tau}_{4}\right) E\right) \\
& +2 \tilde{\tau}_{3}\left(C_{2}-2 t_{2}^{m-2} E\right)-\tilde{\tau}_{4} D \\
& +z\left(\frac{f \tau_{2}}{2}\left(C_{1}+D\right)+f\left(\tau_{1}-\tilde{\tau}_{3}\right) E-\frac{\tilde{f} \tau_{2}}{2}\left(C_{1}-D\right)-\tilde{f}\left(\tau_{1}+\tilde{\tau}_{3}\right) E\right),
\end{aligned}
$$

where we used relations (45) and (46). The above relation implies that $\tilde{\tau}_{3}(0)=\tilde{\tau}_{4}(0)=0$. Therefore, we can write $\tilde{\tau}_{i}(z)=z \tau_{i}(z)$ where $\left.\tau_{i} \in \mathbb{C}\left[t_{2}, z\right]\right](i=3,4)$. The coefficients of $C_{2}$ and $D$ in (67) determine $\tau_{3}$ and $\tau_{4}$ in terms of $\tau_{2}$ :

$$
\begin{gathered}
\tau_{3}=-\frac{1}{2} \partial_{2} \tau_{2}, \\
\tau_{4}=\frac{\tau_{2}}{2}(f+\tilde{f})-\frac{z}{2} \partial_{2}^{2} \tau_{2} .
\end{gathered}
$$


The coefficients of $C_{1}$ and $E$ in (67) give for $\tau_{1}$ and $\tau_{2}$ the system of differential equations

$$
\begin{aligned}
& \partial_{2} \tau_{1}+\frac{\tau_{2}}{2}(f-\tilde{f})=0, \\
& t_{2}^{m-3}\left((m-2)+2 t_{2} \partial_{2}\right)\left(\tau_{2}\right)+\tau_{1}(f-\tilde{f}) \\
& \quad+\frac{z}{2}\left(\partial_{2}\left(\tau_{2}(f+\tilde{f})\right)-z \partial_{2}^{3} \tau_{2}+(f+\tilde{f}) \partial_{2} \tau_{2}\right)=0 .
\end{aligned}
$$

Now suppose that $\left.f \in \mathbb{C}\left\{t_{2}, z\right]\right]$ is given. We claim that there exist solutions $\left(\tau_{1}, \tau_{2}, \tilde{f}\right)$ of (70), (71) with $\left.\tau_{1}, \tau_{2} \in \mathbb{C}\left\{t_{2}, z\right]\right],\left.\tau_{1}\right|_{t_{2}=0} \in \mathbb{C}[[z]]^{*}$ arbitrary and $\tilde{f} \in \mathbb{C}[[z]]\left[t_{2}\right]_{\leq m-4}$ if $m \geq 4$, respectively, $\tilde{f}=0$ if $m=3$. We only prove the statement for $m \geq 4$ (the statement for $m=3$ can be proved similarly). We write $f=\sum_{n \geq 0} f(n) z^{n}$ with $f(n) \in \mathbb{C}\left\{t_{2}\right\}$, and similarly $\tau_{i}=\sum_{n \geq 0} \tau_{i}(n) z^{n}(i \in\{1,2\}), \tilde{f}=\sum_{n \geq 0} \tilde{f}(n) z^{n}$ where $\tau_{i}(n)$ and $\tilde{f}(n)$ depend only on $t_{2}$. Let $\Delta$ be an open disk centered at $0 \in \mathbb{C}$ with $f(n) \in \mathcal{O}_{\Delta}$ for all $n \geq 0$.

Relations (70), (71) give for $\tau_{1}(0), \tau_{2}(0)$ and $\tilde{f}(0)$ the system of equations

$$
\begin{gathered}
\frac{\mathrm{d}}{\mathrm{d} t_{2}} \tau_{1}(0)+\frac{1}{2} \tau_{2}(0)(f(0)-\tilde{f}(0))=0, \\
t_{2}^{m-3}\left((m-2)+2 t_{2} \frac{\mathrm{d}}{\mathrm{d} t_{2}}\right)\left(\tau_{2}(0)\right)+\tau_{1}(0)(f(0)-\tilde{f}(0))=0 .
\end{gathered}
$$

As $\tau_{1}(0)(0) \in \mathbb{C}^{*}$, solvability of (73) requires that $t_{2}^{m-3}$ divides $f(0)-\tilde{f}(0)$. Since $\tilde{f}(0) \in$ $\mathbb{C}\left[t_{2}\right]_{\leq m-4}$, we obtain $\tilde{f}(0)=[f(0)]_{\leq m-4}$. Therefore, $f(0)-\tilde{f}(0)=[f(0)]_{\geq m-3}$. After dividing (73) by $t_{2}^{m-3}$, the system (72), (73) takes the form (40), (41). Using Lemma 14, we obtain, for each value $\tau_{1}(0)(0) \in \mathbb{C}^{*}$, a unique formal solution $\left(\tau_{1}(0), \tau_{2}(0)\right)$. This solution is holomorphic on $\Delta$.

Consider now $n \geq 1$ and assume that $\tau_{1}(k), \tau_{2}(k) \in \mathcal{O}_{\Delta}$ and $\tilde{f}(k) \in \mathbb{C}\left[t_{2}\right]_{\leq m-4}$ are known, for any $k \leq n-1$, such that (70), (71) hold up to order $n-1$. The coefficients of $z^{n}$ in (70), (71) give for $\tau_{1}(n), \tau_{2}(n)$ and $\tilde{f}(n)$ the system of equations

$$
\begin{aligned}
& \frac{\mathrm{d}}{\mathrm{d} t_{2}} \tau_{1}(n)+\frac{1}{2} \tau_{2}(n)[f(0)]_{\geq m-3}+\frac{1}{2} \tau_{2}(0)(f(n)-\tilde{f}(n))+h_{1}(n)=0 \\
& t_{2}^{m-3}\left((m-2)+2 t_{2} \frac{\mathrm{d}}{\mathrm{d} t_{2}}\right)\left(\tau_{2}(n)\right)+\tau_{1}(0)(f(n)-\tilde{f}(n))+\tau_{1}(n)[f(0)]_{\geq m-3} \\
& \quad+h_{2}(n)=0,
\end{aligned}
$$

where $h_{1}(n), h_{2}(n) \in \mathcal{O}_{\Delta}$ are known functions, which depend on $\tau_{1}(k), \tau_{2}(k)$ and $\tilde{f}(k)$ for $k \leq n-1$. Solvability of (75) requires that $t_{2}^{m-3}$ divides $\tau_{1}(0)(f(n)-\tilde{f}(n))+h_{2}(n)$. Let $\tilde{f}(n) \in \mathbb{C}\left[t_{2}\right]_{\leq m-4}$ be the unique polynomial of degree at most $m-4$ such that this property holds. With this definition of $\tilde{f}(n)$, Eqs. (74), (75) reduce [after dividing (75) by $t_{2}^{m-3}$ ], to the system of equations

$$
\begin{gathered}
\frac{\mathrm{d}}{\mathrm{d} t_{2}} \tau_{1}(n)+\frac{1}{2} \tau_{2}(n)[f(0)]_{\geq m-3}+h_{3}(n)=0 \\
\left((m-2)+2 t_{2} \frac{\mathrm{d}}{\mathrm{d} t_{2}}\right)\left(\tau_{2}(n)\right)+\tau_{1}(n)[f(0)]_{\geq m-3} t_{2}^{-(m-3)}+h_{4}(n)=0,
\end{gathered}
$$

where $h_{3}(n), h_{4}(n) \in \mathcal{O}_{\Delta}$ are known. Lemma 14 applies to the system (76), (77) and gives, for each value $\tau_{1}(n)(0) \in \mathbb{C}$, a unique solution $\left(\tau_{1}(n), \tau_{2}(n)\right)$ which is holomorphic on $\Delta$. The existence of a solution $\left(\tau_{1}, \tau_{2}, \tilde{f}\right)$ for (74), (75) with $\left.\tau_{i} \in \mathbb{C}\left\{t_{2}, z\right]\right]$ and $\tilde{f} \in \mathbb{C}[[z]]\left[t_{2}\right]_{\leq m-4}$ follows by induction. 
It remains to prove the uniqueness part of claim (iii). Suppose that $\left(\tau_{1}, \tau_{2}, f, \tilde{f}\right)$ satisfy (70), (71) and $\left.\tau_{1}, \tau_{2} \in \mathbb{C}\left\{t_{2}, z\right]\right], f, \tilde{f} \in \mathbb{C}[[z]]\left[t_{2}\right]_{\leq m-4}$. We need to prove that $f=\tilde{f}$. Equation (73) together with $\tau_{1}(0)(0) \neq 0$ and $f(0), \tilde{f}(0) \in \mathbb{C}\left[t_{2}\right]_{\leq m-4}$ implies that $f(0)=$ $\tilde{f}(0)$ and $\tau_{2}(0)=0$. Then, Eq. (72) implies that $\tau_{1}(0) \in \mathbb{C}^{*}$. Consider now $n \geq 1$. Assume that $f(k)=\tilde{f}(k), \tau_{1}(k) \in \mathbb{C}$ and $\tau_{2}(k)=0$ for $k \leq n-1$. As $h_{1}(n)=\frac{1}{2} \sum_{k=1}^{n-1} \tau_{2}(n-$ $k)(f(k)-\tilde{f}(k))$ we obtain that $h_{1}(n)=0$. Similarly, from the definition of $h_{2}$ and $f(k)=$ $\tilde{f}(k), \tau_{2}(k)=0$ for any $k \leq n-1$, we obtain $h_{2}(n)=0$. Equation (75), together with $[f(0)]_{\geq m-3}=0, h_{2}(n)=0$ and $\tau_{1}(0)(0) \in \mathbb{C}^{*}$, implies, as before, that $f(n)=\tilde{f}(n)$ and $\tau_{2}(n)=0$. Equation (74) implies that $\tau_{1}(n) \in \mathbb{C}$. Inductively, we obtain $f=\tilde{f}, \tau_{1} \in \mathbb{C}[[z]]^{*}$ and $\tau_{2}=0$. This finishes the proof of claim (iii).

Remark 17 (i) The germs $I_{2}(m)(m \geq 3)$ coincide with the germs at the origin of the orbit spaces $\mathbb{C}^{2} / W$ of various Coxeter groups $W$, with their natural $F$-manifold structure (see [9], page 19). In particular, $W=A_{2}$ for $I_{2}(3), W=B_{2}=C_{2}$ for $I_{2}(4), W=H_{2}$ for $I_{2}(5)$ and $W=G_{2}$ for $I_{2}(6)$ (see [2,3] for the definition and classification of Coxeter groups and Lecture 4 of [5], reference [6], Theorem 14 of [7], or Theorem 5.18 of [9] for the natural $F$-manifold structure on their orbit spaces). An immediate consequence of Theorem 16 (iii) is that any two formal $(T)$-structures over the germ at the origin of $\mathbb{C}^{2} / A_{2}$ are formally isomorphic.

(ii) The multiplication o of $I_{2}(m)$ underlies a Frobenius manifold structure. This follows from the general fact that the $F$-manifold multiplication of the orbit space of a Coxeter group can be extended to a Frobenius manifold structure (see Lecture 4 of [5] or reference [6]). Therefore, over $I_{2}(m)$ lies a standard $(T)$-structure

$$
\nabla_{X} Y:=D_{X}^{\tilde{g}} Y+\frac{1}{z} X \circ Y, \quad \forall X, Y \in \mathcal{T}_{M},
$$

where $D^{\tilde{g}}$ is the Levi-Civita connection of the Frobenius metric $\tilde{g}=\mathrm{d} t_{1} \otimes \mathrm{d} t_{2}+\mathrm{d} t_{2} \otimes \mathrm{d} t_{1}$. This standard $(T)$-structure coincides with the normal form (47) with $f=0$. Let us consider now a normal form (47) with $f \in \mathbb{C}\left[t_{2}\right]_{\leq m-4}$ (i.e., $f$ independent of $z$ ). It is mapped, by means of the gauge isomorphism

$$
T=\left(\begin{array}{ll}
1 & \beta \\
0 & 1
\end{array}\right)
$$

with $\beta=\beta\left(t_{2}\right) \in \mathbb{C}\left[t_{2}\right]_{\leq m-3}$ such that $\dot{\beta}=-f$, to the $(T)$-structure with

$$
\tilde{A}_{1}=C_{1}, \quad \tilde{A}_{2}=T^{-1} A_{2} T+T^{-1} z \partial_{2} T=\left(\begin{array}{cc}
-\beta & t_{2}^{m-2}-\beta^{2} \\
1 & \beta
\end{array}\right) .
$$

Remark that both $\tilde{A}_{1}$ and $\tilde{A}_{2}$ in (79) are independent on $z$. This is an example of a general result, namely that any $(T)$-structure is locally (holomorphically) isomorphic to a $(T)$-structure with connection form $\frac{1}{z} \sum_{i} \tilde{A}_{i} \mathrm{~d} t_{i}$ where $\tilde{A}_{i}=\tilde{A}_{i}(0)$ are independent on $z$. We shall prove this result in a forthcoming paper.

\section{$5(T)$-structures over $\mathcal{N}_{2}$}

In this section, we find formal normal forms for $(T)$-structures over $\mathcal{N}_{2}$. In a first stage, we will find them up to formal gauge isomorphisms. They are described in Theorem 18, whose proof relies on the calculations from the proof of Theorem 16. In a second stage, we 
will exploit the additional freedom from holomorphic isomorphisms which lift non-trivial automorphisms of the base. Theorem 19 states the results. Finally, Theorem 21 combines Theorems 18 and 19 and gives round formal normal forms for $(T)$-structures over $\mathcal{N}_{2}$ up to the entire group of formal isomorphisms.

We will use the same matrices $C_{1}, D$ and $E$, as in the previous section. The definition of the matrix $C_{2}$ is almost the same as before, with the only difference that the $(1,2)$ entry $t_{2}^{m-2}$ is replaced by 0 . Thus,

$$
C_{1}:=\mathrm{Id}_{2}, \quad C_{2}:=\left(\begin{array}{ll}
0 & 0 \\
1 & 0
\end{array}\right), \quad D:=\left(\begin{array}{cc}
1 & 0 \\
0 & -1
\end{array}\right), \quad E:=\left(\begin{array}{ll}
0 & 1 \\
0 & 0
\end{array}\right) .
$$

Relations (45) and (46) still hold, with $t_{2}^{m-2}$ replaced by 0 .

Theorem 18 (i) Over $\mathcal{N}_{2}$, any (T)-structure is gauge isomorphic to a (T)-structure of the form (47) where $f \in \mathbb{C}\left\{t_{2}, z\right\}$ is holomorphic.

(ii) Any formal $(T)$-structure over $\mathcal{N}_{2}$ is formally gauge isomorphic to a formal $(T)$ structure of the form (47) where $\left.f \in \mathbb{C}\left\{t_{2}, z\right]\right]$.

(iii) For a holomorphic or formal (T)-structure of the form (47) over $\mathcal{N}_{2}$, with $f \in \mathbb{C}\left\{t_{2}, z\right\}$, respectively, $\left.f \in \mathbb{C}\left\{t_{2}, z\right]\right]$, the function $f(0) \in \mathbb{C}\left\{t_{2}\right\}$ is a formal gauge invariant of it. If $f(0)=0$, then also the function $f(1) \in \mathbb{C}\left\{t_{2}\right\}$ is a formal gauge invariant of it.

(iv) A holomorphic (T)-structure of the form (47) over $\mathcal{N}_{2}$, with $f(0)=0$, is gauge isomorphic to a unique $(T)$-structure of the form

$$
A_{1}=C_{1}, \quad A_{2}=C_{2}+z^{2} a(1) E .
$$

where $a(1) \in \mathbb{C}\left\{t_{2}\right\}$. The coefficient a(1) coincides with $f(1)$.

(v) A holomorphic or formal (T)-structure of the form (47) with $f(0) \neq 0$ and $\operatorname{ord}_{0} f(0)=$ $r \in \mathbb{Z}_{\geq 0}$ is formally gauge isomorphic to the $(T)$-structure

$$
A_{1}=C_{1}, \quad A_{2}=C_{2}+z a(0) E
$$

if $r \in\{0,1\}$, or to a formal (T)-structure

$$
A_{1}=C_{1}, \quad A_{2}=C_{2}+z \tilde{f} E
$$

where $\tilde{f}(0)=f(0)$ and $\tilde{f}(k) \in \mathbb{C}\left[t_{2}\right]_{\leq r-2}$, for any $k \geq 1$, if $r \geq 2$. These normal forms are formal gauge invariants and are unique.

Proof Claims (i) and (ii) follow with the same argument as steps 1-3 from the proof of Theorem 16. [The only difference lies in relations (62) and (63), in which the terms containing with $t_{2}^{m-3}$ are now replaced by 0 .]

To prove claim (iii), we consider two formal normal forms $A_{1}, A_{2}$ and $\tilde{A}_{1}, \tilde{A}_{2}$ as in (64) and a matrix $T=\tau_{1} C_{1}+\tau_{2} T_{2}+\tilde{\tau_{3}} D+\tilde{\tau_{4}} E$ as in (65) such that relation (66) is satisfied (i.e., the formal normal forms are formally gauge isomorphic). We find again that $T$ is independent on $t_{1}, \tilde{\tau_{3}}(0)=0, \tilde{\tau_{4}}(0)=0$ and thus write again $\tilde{\tau_{3}}(z)=z \tau_{3}(z)$ and $\tilde{\tau_{4}}(z)=z \tau_{4}(z)$ with $\tau_{3}$, $\left.\tau_{4} \in \mathbb{C}\left\{t_{2}, z\right]\right]$. The same calculations lead to the same Eqs. (68), (69), which determine $\tau_{3}$ and $\tau_{4}$ in terms of $\tau_{2}, f, \tilde{f}$, and to the system of equations

$$
\begin{gathered}
\partial_{2} \tau_{1}+\frac{\tau_{2}}{2}(f-\tilde{f})=0, \\
\tau_{1}(f-\tilde{f})+\frac{z}{2}\left(\partial_{2}\left(\tau_{2}(f+\tilde{f})\right)-z \partial_{2}^{3} \tau_{2}+(f+\tilde{f}) \partial_{2} \tau_{2}\right)=0 .
\end{gathered}
$$

As $\left.\tau_{1} \in \mathbb{C}\left\{t_{2}, z\right]\right]$ is invertible, relation (85) implies that $f(0)=\tilde{f}(0)$. We obtain that $f(0)$ is a formal gauge invariant, as needed. Suppose now that $f(0)=\tilde{f}(0)=0$. Identifying 
the coefficient of $z$ in (85) we obtain $\tau_{1}(0)(f(1)-\tilde{f}(1))=0$. Since $\tau_{1}(0) \in \mathbb{C}\left\{t_{2}\right\}$ is a unit, we deduce that $f(1)=\tilde{f}(1)$, i.e., $f(1)$ is a formal gauge invariant. The proof of claim (iii) is completed.

We now prove claim (iv). For this, we consider a $(T)$-structure of the form (47) with $f \in \mathbb{C}\left\{t_{2}, z\right\}$ and $f(0)=0$. Define $\tilde{f}:=z f(1)$. We claim that the system of differential equations (84), (85) has a holomorphic solution $\left(\tau_{1}, \tau_{2}\right) \in \mathbb{C}\left\{t_{2}, z\right\}^{2}$ with $\left.\tau_{1}\right|_{t_{2}=0} \in \mathbb{C}\{z\}^{*}$ arbitrary. Write $f-\tilde{f}=z^{2} g$ with $g \in \mathbb{C}\left\{t_{2}, z\right\}$. Then, (84), (85) become the system

$$
\begin{gathered}
\partial_{2} \tau_{1}+\frac{1}{2} z^{2} \tau_{2} g=0, \\
\partial_{2}^{3} \tau_{2}-2 \tau_{1} g-\partial_{2}\left(\tau_{2}(2 f(1)+z g)\right)-(2 f(1)+z g) \partial_{2} \tau_{2}=0,
\end{gathered}
$$

with leading parts $\partial_{2} \tau_{1}$ and $\partial_{2}^{3} \tau_{2}$. It can be rewritten as a system of linear differential equations in $t_{2}$ with holomorphic parameter $z$, and it has a holomorphic solution $\left(\tau_{1}, \tau_{2}\right)$ with $\left.\tau_{1}\right|_{t_{2}=0} \in$ $\mathbb{C}\{z\}^{*}$ arbitrary. Claim (iv) is proved. [The uniqueness follows from claim (iii).]

To prove claim (v), we consider a holomorphic or formal $(T)$-structure of the form (47) over $\mathcal{N}_{2}$ with $\left.f \in \mathbb{C}\left\{t_{2}, z\right]\right]$ and $\operatorname{ord}_{0} f(0)=r \in \mathbb{Z}_{\geq 0}$. We need to show that there exists a solution $\left(\tau_{1}, \tau_{2}, \tilde{f}\right)$ of $(84)$, (85) with $\left.\tau_{1}, \tau_{2} \in \mathbb{C}\left\{t_{2}, z\right]\right],\left.\tau_{1}\right|_{t_{2}=0} \in \mathbb{C}[[z]]^{*}$ arbitrary and $\tilde{f}=f(0)$ if $r \in\{0,1\}$ and $\tilde{f}(0)=f(0), \tilde{f}(k) \in \mathbb{C}\left[t_{2}\right]_{\leq r-2}$ for $k \geq 1$, if $r \geq 2$. Let $\Delta$ be an open disk centered at $0 \in \mathbb{C}$ with $f(k) \in \mathcal{O}_{\Delta}$ for all $k \geq 0$.

Equations (84), (85) give for $\tau_{1}(0), \tau_{2}(0)$ and $\tilde{f}(1)$ the equations

$$
\frac{\mathrm{d}}{\mathrm{d} t_{2}} \tau_{1}(0)=0, \quad \tau_{1}(0)(f(1)-\tilde{f}(1))+\frac{\mathrm{d}}{\mathrm{d} t_{2}}\left(\tau_{2}(0) f(0)\right)+f(0) \frac{\mathrm{d}}{\mathrm{d} t_{2}} \tau_{2}(0)=0 .
$$

Choose $\tau_{1}(0) \in \mathbb{C}^{*}$ arbitrary. Then, for any $\tilde{f}(1)$ fixed, the second relation (88) can be considered as a differential equation of the form (36) in the unknown function $\tau_{2}(0)$. When $r \in\{0,1\}$, we define $\tilde{f}(1):=0$. When $r \geq 2$, we define $\tilde{f}(1) \in \mathbb{C}\left[t_{2}\right]_{\leq r-2}$ to be the unique polynomial of degree at most $r-2$ such that $t_{2}^{r-1}$ divides $f(1)-\tilde{f}(1)$. In both cases, Lemma 12 provides a holomorphic solution $\tau_{2}(0) \in \mathcal{O}_{\Delta}$.

Let $n \geq 1$. When $r \geq 2$, suppose that $\tau_{1}(k), \tau_{2}(k) \in \mathcal{O}_{\Delta}, \tilde{f}(k+1) \in \mathbb{C}\left[t_{2}\right]_{\leq r-2}$ $(0 \leq k \leq n-1)$ have been constructed such that Eq. (84) up to order $n-1$ in $z$ and Eq. (85) up to order $n$ in $z$ are satisfied. When $r \in\{0,1\}$, suppose that $\tau_{1}(k), \tau_{2}(k) \in \mathcal{O}_{\Delta}$ $(0 \leq k \leq n-1)$ have been constructed such that Eq. (84) up to order $n-1$ in $z$ and Eq. (85) up to order $n$ in $z$ are satisfied, with $\tilde{f}(k)=0(1 \leq k \leq n)$. Then, the coefficient of $z^{n}$ in (84) and the coefficient in $z^{n+1}$ of (85) give for $\tau_{1}(n), \tau_{2}(n)$ and $\tilde{f}(n+1)$ the equations

$$
\begin{aligned}
& \frac{\mathrm{d}}{\mathrm{d} t_{2}} \tau_{1}(n)+h_{1}(n)=0, \\
& \frac{\mathrm{d}}{\mathrm{d} t_{2}}\left(\tau_{2}(n) f(0)\right)+f(0) \frac{\mathrm{d}}{\mathrm{d} t_{2}} \tau_{2}(n) \\
& \quad+\tau_{1}(n)(f(1)-\tilde{f}(1))+\tau_{1}(0)(f(n+1)-\tilde{f}(n+1))+h_{2}(n)=0,
\end{aligned}
$$

where $h_{1}(n), h_{2}(n) \in \mathcal{O}_{\Delta}$ are known. Let $\tau_{1}(n)$ be a solution of (89). With this choice of $\tau_{1}(n)$, Eq. (90) in the unknown function $\tau_{2}(n)$ becomes

$$
\frac{\mathrm{d}}{\mathrm{d} t_{2}}\left(\tau_{2}(n) f(0)\right)+f(0) \frac{\mathrm{d}}{\mathrm{d} t_{2}} \tau_{2}(n)-\tau_{1}(0) \tilde{f}(n+1)+h_{3}(n)=0,
$$

where $h_{3}(n) \in \mathcal{O}_{\Delta}$ is known. Remark that (91) is of the form (36). From Lemma 12, Eq. (91), with any given $\tilde{f}(n+1)$, has a solution, which is holomorphic on $\Delta$, when $r \in\{0,1\}$. We choose $\tau_{2}(n)$ to be a solution of (91) with $\tilde{f}(n+1):=0$. When $r \geq 2$, Eq. (91) has a solution 
if and only if $t_{2}^{r-1}$ divides $\left(\tau_{1}(0) \tilde{f}(n+1)-h_{3}(n)\right)$. We choose $\tilde{f}(n+1) \in \mathbb{C}\left[t_{2}\right]_{\leq r-2}$ to be the unique polynomial of degree at most $r-2$, such that this property is satisfied, and $\tau_{2}(n) \in \mathcal{O}_{\Delta}$ to be the unique solution of Eq. (91) with this choice of $\tilde{f}(n+1)$. The first statement of claim (v) is proved.

It remains to prove the uniqueness of the normal form. When $r \in\{0,1\}$ this follows from claim (iii). Suppose now that $r \geq 2$. Consider two normal forms of type (83), with functions $f$ and $\tilde{f}$. From claim (iii), $f(0)=\tilde{f}(0)$. Let $\left.\tau_{1} \in \mathbb{C}\left\{t_{2}, z\right]\right]^{*}$ and $\left.\tau_{2} \in \mathbb{C}\left\{t_{2}, z\right]\right]$ which satisfy (84), (85). Going again through the above proof for the existence of the normal form, we find inductively that $\tau_{1}(n) \in \mathbb{C}, \tau_{2}(n)=0$ and $f(n+1)=\tilde{f}(n+1)$ for any $n \geq 0$. The details are as in the proof of Theorem 16 (iii).

Below a function $f \in \mathbb{C}\left\{t_{2}, z\right\}$ is called associated to a $(T)$-structure over $\mathcal{N}_{2}$ if the $(T)$ structure is (holomorphically) isomorphic to the (T)-structure $A_{1}=C_{1}, A_{2}=C_{2}+z f E$. From Theorem 18 (i), any ( $T$ )-structure admits a (non-unique) associated function, which was obtained using gauge isomorphisms. In the next theorem, we will exploit the additional freedom provided by isomorphisms which lift non-trivial automorphisms of $\mathcal{N}_{2}$, in order to simplify the lower-order terms of associated functions.

Theorem 19 Consider an arbitrary $(T)$-structure $(H, \nabla)$ over $\mathcal{N}_{2}$.

(i) The order $\operatorname{ord}_{0} f(0) \in \mathbb{Z}_{\geq 0} \cup\{\infty\}$ of an associated function $f$ is a formal invariant of $(H, \nabla)$ (with $\operatorname{ord}_{0} f(0):=\infty$ when $\left.f(0)=0\right)$.

(ii) If some associated function $\tilde{f}$ of $(H, \nabla)$ satisfies $\tilde{f}(0)=0$, then there is an associated function $f$ with $f(0)=0$ and $f(1)=0$.

(iii) If the order of an associated function $\tilde{f}$ of $(H, \nabla)$ is $r=\operatorname{ord}_{0} \tilde{f}(0) \in \mathbb{Z}_{\geq 0}$, then there is an associated function $f$ with $f(0)=t_{2}^{r}$.

Proof To prove claim (i), we consider two $(T)$-structures, given by

$$
\begin{aligned}
A_{1} & =C_{1}, \quad A_{2}=C_{2}+z f E \\
\tilde{A_{1}} & =C_{1}, \quad \tilde{A_{2}}=C_{2}+z \tilde{f} E
\end{aligned}
$$

with $f, \tilde{f} \in \mathbb{C}\left\{t_{2}, z\right\}$, an automorphism $h:\left(\mathbb{C}^{2}, 0\right) \rightarrow\left(\mathbb{C}^{2}, 0\right)$ of $\mathcal{N}_{2}$ and a matrix $\tilde{T} \in$ $\left.G L_{2}\left(\mathbb{C}\left\{t_{2}, z\right]\right]\right)$ such that relation (12) is satisfied. We will make relations in (12) explicit. From Lemma 11 (ii), $h$ is of the form $h\left(t_{1}, t_{2}\right)=\left(t_{1}, \lambda\left(t_{2}\right)\right)$ with $\lambda \in t_{2} \mathbb{C}\left\{t_{2}\right\}^{*}$. We write

$$
\tilde{T}=\left(\begin{array}{ll}
a & \tilde{e} \\
c & b
\end{array}\right)
$$

with $a, b, c, \tilde{e} \in \mathbb{C}\{t, z]]$. Relations (12) become

$$
z \partial_{j} \tilde{T}+\delta_{j 1} \tilde{T}+\delta_{j 2} \dot{\lambda}\left(A_{2} \circ \lambda\right) \tilde{T}-\tilde{T} \tilde{A_{j}}=0, \quad j \in\{1,2\} .
$$

For $j=1$ relation (94) gives $\left.a, b, c, \tilde{e} \in \mathbb{C}\left\{t_{2}, z\right]\right]$. For $j=2$ it gives

$$
\begin{aligned}
0 & =\left(\begin{array}{cc}
z \partial_{2} a & z \partial_{2} \tilde{e} \\
z \partial_{2} c & z \partial_{2} b
\end{array}\right)+\left(\begin{array}{cc}
0 & z \dot{\lambda}(f \circ \lambda) \\
\dot{\lambda} & 0
\end{array}\right)\left(\begin{array}{ll}
a & \tilde{e} \\
c & b
\end{array}\right)-\left(\begin{array}{ll}
a & \tilde{e} \\
c & b
\end{array}\right)\left(\begin{array}{cc}
0 & z \tilde{f} \\
1 & 0
\end{array}\right) \\
& =\left(\begin{array}{cc}
z\left(\partial_{2} a+\dot{\lambda} c(f \circ \lambda)\right)-\tilde{e} & z\left(\partial_{2} \tilde{e}+\dot{\lambda} b(f \circ \lambda)-\tilde{f} a\right) \\
z \partial_{2} c+\dot{\lambda} a-b & z \partial_{2} b+\dot{\lambda} \tilde{e}-z \tilde{f} c
\end{array}\right) .
\end{aligned}
$$

The $(1,1)$-entry in the above matrix implies that $\tilde{e}(z)=z e(z)$ for $\left.e \in \mathbb{C}\left[t_{2}, z\right]\right]$. As $\tilde{T}$ is invertible, we deduce that $a(0)(0), b(0)(0) \in \mathbb{C}^{*}$. The $(1,2)$ entry in $(95)$ gives

$$
\dot{\lambda} b(0)(f(0) \circ \lambda)=\tilde{f}(0) a(0),
$$


which implies $\operatorname{ord}_{0} f(0)=\operatorname{ord}_{0} \tilde{f}(0)$. Claim (i) is proved.

To prove claims (ii) and (iii), we start with $\tilde{f} \in \mathbb{C}\left\{t_{2}, z\right\}$ as in the assumptions of these claims. We will find $a, b, c, e, f \in \mathbb{C}\left\{t_{2}, z\right\}$ which satisfy (95) (with $\tilde{e}:=z e$ ) and such that $f$ is in the form required by these claims. In both cases, $a \in \mathbb{C}\left\{t_{2}\right\}^{*}$ will be suitably chosen, independent on $z$,

$$
b:=a^{-1} \in \mathbb{C}\left\{t_{2}\right\}^{*}, \quad c:=0, \quad e:=\dot{a} \in \mathbb{C}\left\{t_{2}\right\}
$$

and $\lambda \in t_{2} \mathbb{C}\left\{t_{2}\right\}^{*}$ satisfies

$$
\dot{\lambda}=a^{-2} \text {. }
$$

With these choices, three of the four relations (95) are satisfied. The remaining relation is given by the $(1,2)$ entry of the matrix and is equivalent to

$$
z \ddot{a}+a^{-3}(f \circ \lambda)-\tilde{f} a=0 .
$$

To prove claim (ii), assume that $\tilde{f}(0)=0$ and choose $a$ such that

$$
\ddot{a}=\tilde{f}(1) a .
$$

Then, (99) has a unique solution $f \in \mathbb{C}\left\{t_{2}, z\right\}$ with $f(0)=f(1)=0$ and $f(k) \circ \lambda=\tilde{f}(k) a^{4}$ for $k \geq 2$. Claim (ii) is proved.

To prove claim (iii), assume that $\operatorname{ord}_{0} \tilde{f}(0)=r \in \mathbb{Z}_{\geq 0}$. We start with a solution $\lambda \in$ $t_{2} \mathbb{C}\left\{t_{2}\right\}^{*}$ of the equation

$$
\lambda^{r}(\dot{\lambda})^{2}=\tilde{f}(0)
$$

(which exists from Lemma 13). Then, we choose $a \in \mathbb{C}\left\{t_{2}\right\}^{*}$ such that (98) holds, and then $b, c, e$ as in (97). The function $f \in \mathbb{C}\left\{t_{2}, z\right\}$ with $f(0)=t_{2}^{r}, f(1) \circ \lambda=\tilde{f}(1) a^{4}-a^{3} \ddot{a}$ and $f(k) \circ \lambda=\tilde{f}(k) a^{4}$, for $k \geq 2$, satisfies (99). Claim (iii) is proved.

Remark 20 The notion of an associated function can be extended to formal $(T)$-structures over $\mathcal{N}_{2}$, by replacing in their definition '(holomorphically) isomorphic' with 'formally isomorphic.' For formal $(T)$-structures, associated functions belong to $\left.\mathbb{C}\left\{t_{2}, z\right]\right]$. Theorem 18 (ii) shows that any formal $(T)$-structure admits an associated function. Theorem 19 holds also for formal $(T)$-structures.

Our main result from this section is the next theorem, which states the formal classification of $(T)$-structures over $\mathcal{N}_{2}$.

Theorem 21 (i) Any (T)-structure (or formal (T)-structure) over $\mathcal{N}_{2}$ is formally isomorphic to a $(T)$-structure of the form

$$
\begin{aligned}
& A_{1}=C_{1}, \quad A_{2}=C_{2}+z E \\
& A_{1}=C_{1}, \quad A_{2}=C_{2}+z t_{2} E \\
& A_{1}=C_{1}, \quad A_{2}=C_{2},
\end{aligned}
$$

or to a holomorphic or formal (T)-structure of the form

$$
A_{1}=C_{1}, \quad A_{2}=C_{2}+z\left(t_{2}^{r}+\sum_{k \geq 1} f(k) z^{k}\right) E,
$$

where $f(k) \in \mathbb{C}\left[t_{2}\right]_{\leq r-2}$ are polynomials of degree at most $r-2$ and $r \in \mathbb{Z}_{\geq 2}$. 
(ii) Any two different ( $T$ )-structures (or formal ( $T$ )-structures) $\nabla$ and $\tilde{\nabla}$ from (i), at least one of them not being of the form (105), are formally non-isomorphic. If $\nabla$ and $\tilde{\nabla}$ are of the form (105), then they are formally gauge non-isomorphic. They are formally isomorphic if and only if there is $\lambda_{0} \in \mathbb{C}, \lambda_{0}^{r}=1$, such that $\tilde{f}(k)\left(t_{2}\right)=\lambda_{0}^{-2} f(k)\left(\frac{t_{2}}{\lambda_{0}}\right)$, for any $k \geq 1$.

Proof We only prove the statements for $(T)$-structures (the arguments for formal $(T)$ structures are similar). Let $\nabla$ be a $(T)$-structure over $\mathcal{N}_{2}$ and $\tilde{f}$ an associated function. If $\tilde{f}(0) \neq 0$, then, using Theorem 19 (iii), we can assume that $\tilde{f}(0)=t_{2}^{r}$ with $r \in \mathbb{Z}_{\geq 0}$. Then, Theorem $18(\mathrm{v})$ implies that $\nabla$ is formally isomorphic to a $(T)$-structure of the form (102), (103) or to a $(T)$-structure or formal $(T)$-structure of the form (105). If $\tilde{f}(0)=0$, then, using Theorem 19 (ii), we can assume that $\tilde{f}(1)=0$. Then, Theorem 18 (iv) implies that $\nabla$ is formally isomorphic to the (T)-structure (104). Claim (i) is proved.

The first two parts of claim (ii) follow from Theorem 19 (i) together with the uniqueness part in Theorem 18 (v). Assume now that $\nabla$ and $\tilde{\nabla}$ are two formally isomorphic $(T)$-structures of the form (105). Let $T$ be a formal isomorphism between them. It covers a map of the form $h\left(t_{1}, t_{2}\right)=\left(t_{1}, \lambda\left(t_{2}\right)\right)$, with $\lambda(0)=0$ and $\dot{\lambda}(0) \neq 0$. From relation (12) together with $A_{2}(0)=\tilde{A}_{2}(0)=C_{2}$ and $f(0)=\tilde{f}(0)=t_{2}^{r}$, we deduce that $\lambda$ satisfies $(\dot{\lambda})^{2} \lambda^{r}=t_{2}^{r}$. From Lemma $13, \lambda\left(t_{2}\right)=\lambda_{0} t_{2}$, where $\lambda_{0}^{r}=1$. Consider now the isomorphism $T_{1}$ which covers $h$ and is given by the constant matrix $\operatorname{diag}\left(1, \lambda_{0}\right)$. Then, $\nabla^{(1)}:=T_{1} \cdot \nabla$ is a $(T)$-structure with $A_{1}^{(1)}=C_{1}, A_{2}^{(1)}=C_{2}+z f^{(1)} E$, where $f^{(1)}(0)=t_{2}^{r}$ and $f^{(1)}(k)\left(t_{2}\right)=\lambda_{0}^{-2} f(k)\left(\frac{t_{2}}{\lambda_{0}}\right)$, for any $k \geq 1$. Remark that $\nabla^{(1)}$ and $\tilde{\nabla}$ are formally gauge isomorphic (by means of the formal gauge isomorphism $\left.T \circ T_{1}^{-1}\right)$. Therefore, they coincide. We deduce that $\tilde{f}=f^{(1)}$, which completes the proof of claim (ii).

Remark 22 It is natural to ask if a ( $T)$-structure is holomorphically isomorphic to its formal normal form provided by Theorems 16 (iii) or 21 . We believe that this is not, in general, true. Let us consider the $(T)$-structures over $I_{2}(m)$, with $m \geq 4$. We do not believe that the functions $\tau_{1}, \tau_{2}$ and $\tilde{f}$ constructed in the proof of part (iii) of Theorem 16 are in general holomorphic if $f$ is holomorphic. Therefore, in order to obtain holomorphic normal forms for $(T)$-structures over $I_{2}(m)$ one needs to modify part (iii) in Theorem 16 substantially. We plan to work on this. We also plan to work on the formal and holomorphic classification of $(T E)$-structures.

Acknowledgements L.D. was supported by a grant of the Ministry of Research and Innovation, CNCSUEFISCDI, Project No. PN-III-P4-ID-PCE-2016-0019 within PNCDI III. Part of this work was done during her visit at University of Mannheim (Germany) in October 2017. She thanks University of Mannheim for hospitality and great working conditions.

\section{References}

1. Anosov, D.V., Bolibruch, A.A.: The Riemann-Hilbert Problem, Aspects of Mathematics, vol. 22. Vieweg, Braunschweig (1994)

2. Bourbaki, N.: Groupes et Algebres de Lie, Chapitres 4, 5 et 6. Hermann, Paris (1968)

3. Coxeter, H.S.M.: Discrete groups generated by reflections. Ann. Math. 35, 588-621 (1934)

4. David, L., Hertling, C.: Regular $F$-manifolds: initial conditions and Frobenius metrics. Ann. Sci. Norm. Super. Pisa Cl. Sci (5) XVII, 1121-1152 (2017)

5. Dubrovin, B.: Geometry of $2 D$ topological field theories. In: Francoviglia, M., Greco, S. (eds.) Integrable Systems and Quantum Groups. Montecatini, Terme 1993, Lecture Notes in Mathematics, vol. 1620, pp. 120-348. Springer, Berlin (1996)

6. Dubrovin, B.: Differential geometry of the space of orbits of a Coxeter group. In: Terng, C.L., Uhlenbeck, K. (eds.) Survey of Differential Geometry [Integrable Systems]. Surveys in Differential Geometry, vol. IV, pp. 181-211. International Press, Boston, MA (1998) 
7. Givental, A.B.: Singular Lagrangian manifolds and their Lagrangian maps. J. Soviet. Math. 52(4), 3246$3278(1998)$

8. Hertling, C.: $t t^{*}$ geometry, Frobenius manifolds, their connections, and the construction for singularities. J. Reine Angew. Math. 555, 77-161 (2003)

9. Hertling, C.: Frobenius Manifolds and Moduli Spaces for Singularities, Cambridge Tracts in Mathematics, vol. 151. Cambridge University Press, Cambridge (2002)

10. Hertling, C., Manin, Y.: Weak Frobenius manifolds. Int. Math. Res. Not. 6, 277-286 (1999)

11. Hertling, C., Manin, Y.: Unfoldings of meromorphic connections and a construction theorem for Frobenius manifolds. In: Hertling, C., Marcolli, M. (eds.) Frobenius Manifolds, Quantum Cohomology, and Singularities, pp. 113-144. Vieweg, Braunschweig (2004)

12. Hertling, C., Manin, Y., Teleman, C.: An update on semisimple quantum cohomology and $F$-manifolds. Proc. Steklov Inst. Math. 264, 62-69 (2009)

13. Hertling, C., Hoevenaars, L., Posthuma, H.: Frobenius manifolds, projective special geometry and Hitchin systems. J. Reine Angew. Math. 649, 117-165 (2010)

14. Lorenzoni, P., Pedroni, M., Raimondo, A.: F-manifolds and integrable systems of hydrodynamic type. Arch. Math. 47, 163-180 (2011)

15. Malgrange, B.: Deformations de systemes differentielles et microdifferentielles. In: Seminaire E.N.S. Mathematique et Physique, pp. 351-379

16. Malgrange, B.: Deformations of differential systems II. J. Ramanjuan Math. Soc. 1, 3-15 (1986)

17. Malgrange, B.: Equations Differentielles a Coefficients Polynomiaux, Progress in Mathematics, vol. 96. Birkhauser, Basel (1991)

18. Sabbah, C.: Isomonodromic Deformations and Frobenius Manifolds. Springer, Berlin (2007)

19. Strachan, : Frobenius manifolds: natural submanifolds and induced bi-Hamiltonian structures. Diff. Geom. Appl. 20, 67-99 (2004)

20. Wasow, W.: Asymptotic Expansions for Ordinary Differential Equations. Dover Publications, Mineola (1965)

Publisher's Note Springer Nature remains neutral with regard to jurisdictional claims in published maps and institutional affiliations. 